\title{
Output-Feedback Control for Path Following of Autonomous Underwater Vehicles via Singular Perturbation Technique
}

Ming Lei ( $\nabla$ shandian2010@hrbeu.edu.cn )

Harbin Engineering University https://orcid.org/0000-0002-0299-2475

Ye Li

Harbin Engineering University

Shuo Pang

Harbin Engineering University

\section{Research Article}

Keywords: Autonomous Underwater Vehicles (AUVs), Path Following, Extended State Observer (ESO), Singular Perturbations, Time Scale Decomposition.

Posted Date: April 12th, 2021

DOl: https://doi.org/10.21203/rs.3.rs-381024/v1

License: (c) (i) This work is licensed under a Creative Commons Attribution 4.0 International License. Read Full License 


\title{
Output-Feedback Control for Path Following of Autonomous Underwater Vehicles via Singular Perturbation Technique
}

\author{
Ming Lei, Ye Li and Shuo Pang \\ Science and Technology on Underwater Vehicle Laboratory, Harbin Engineering University, Harbin 150001, China \\ E-mail: shandian2010@hrbeu.edu.cn \& liyeheu1403@163.com \& pangshuo1403@163.com
}

\begin{abstract}
Autonomous underwater vehicles are needed in many applications such as underwater monitoring and surveillance, marine biology, rescue and search, undersea oil detection. In reality, the unknown external disturbances, and model uncertainties make the motion control of autonomous underwater vehicles a challenging task. With these issues, this paper presents an output-feedback singular perturbation control scheme for the path following of autonomous underwater vehicles, in term of the time scale decomposition method. As illustration, an extended state observer is first devised based on the singular perturbation theory. Then the stabilizing controller is developed by using the time scale decomposition method, in order to obtain a simple, easy-to-implement control law. And the stability analysis of stabilizing control system is conducted by constructing a composite Lyapunov function, which allows to provide mathematical bounds on the design parameters. Finally, simulation results are presented to prove the efficacy of the proposed controller for path following of autonomous underwater vehicles subject to internal and external disturbances.
\end{abstract}

Keywords: Autonomous Underwater Vehicles (AUVs); Path Following; Extended State Observer (ESO); Singular Perturbations; Time Scale Decomposition.

\section{INTRODUCTION}

AUV plays an important role in marine activities such as ocean sampling, resource exploration and exploitation, and rescue and search, and so on, since it provides a safe, efficient, and economical way without placing human lives at risk. To fulfill these deep oceanic missions, effective motion control is essential. In view of the different application scenarios, several fundamental motion control problems, such as path following $[1,3,4,6]$, trajectory tracking [2, 8, 9] and formation tracking [7, 11], are focused. This article considers the path following control of AUVs that finds many applications in reality, such as oceanographic survey, target carpet searching and pipeline inspection.

In practical cases, there are many challenges in the motion control of an AUV, such as complex dynamic behavior, parameter variations, model errors and external disturbances caused by sea currents and waves. All these make the motion control of an AUV attractive to researchers. During the past two decades, a wide range of different nonlinear control techniques have been applied to study this issue and many results are reported. On account of the ability to improve the control performance in case of little or no information of the bounds on uncertainties, the adaptive control becomes a mainstream method for the motion control of AUVs subject to system uncertainties. In [6], a hybrid adaptation scheme is proposed to enhance the robustness against the parametric uncertainty. In [12], a nonlinear robust adaptive control strategy is developed for path following of underactuated AUVs, based on Lyapunov's direct method, back-stepping and parameter projection techniques. In [16], an adaptive switching control scheme is designed for 3D trajectory tracking and path following of underactuated AUVs. The sliding mode control (SMC) is another mainstream 
method for the control of AUVs, due to the insensitivity to model uncertainties and unknown disturbances. In [8], a sliding mode control scheme is proposed for the lateral trajectory tracking of an underactuated AUV. In [9], an trajectory tracking controller is obtained by the combining the back-stepping technique and adaptive dynamical SMC. To eliminate the main drawback associated with the SMC, known as the chattering effect due to the discontinuous control signal, in [10] and [15], two different higher order sliding mode control schemes are introduced.

Since Fuzzy logic systems (FLS) and Neural Networks (NN) are capable to approximate nonlinearities, as a result, provide improved robustness properties under model uncertainties and unknown disturbances, they have been widely used in the control system design. In [5], anti-disturbance constrained control of AUVs subject to uncertainties and constraints is developed, using $\mathrm{NN}$ approach and observer-based technique. In [14], a robust fuzzy control law is proposed for 3D path following of AUVs. In [17], an adaptive output feedback controller is designed for the 3D trajectory tracking of AUVs, based on dynamic recurrent fuzzy neural network (DRFNN). As illustration, all these nonlinear control techniques mentioned above have the advantages on addressing the technical challenges of model uncertainties and unknown external disturbances. However, they usually yield relatively complicated controllers which may be prohibitive in the real world.

As a simple but effective tool for dealing with the unknown disturbances of complex system, the observer-based control technique has also drawn obvious attentions of researchers for the control system design given unknown external and internal disturbances. In [3] and [4], two controllers with different focuses are developed for path-following of underactuated AUVs based on ESO and NN. In [18], a high-gain observer-based output feedback control law is employed for remotely operated vehicles (ROVs), considering the model errors, measurement errors, and unknown external environmental disturbances.

Theory of singular perturbation and time scale represents a mathematical realization of intuitive approach to simplified models obtained via order reduction $[19,20]$. Compared to most classical control methods, the distinct feature of singular perturbation control is that it allows a time scale decomposition of a dynamic system into lower order subsystems with different time scales, in which the control laws can be designed independently and thus being easily obtained, leading to a reduction of control complexity. Such characteristic is deeply appealing to researchers and control engineers. It has been widely applied to the design and analysis of motion control of marine vehicles. In [21], a robust nonlinear feedback control law is developed for ROVs equipped with a robot manipulator, by utilizing the difference between the two-time operation scale between the vehicle and the manipulator. In [22], several Lyapunov-based controllers are proposed for wing-level flight of underwater gliders with different configurations. In [23], a nonlinear, passivity-based controller and a observer is designed for a gliding robotic fish, based on the existing reduced subsystem as in [22]. In [24] and [25], two distinct two-time scale singular perturbation control laws are employed for rudder roll stabilization (RRS) and path following of marine surface vessels, respectively. In [26], a simple inner-and-outer loop PD controller is proposed for the pitch-depth diving motion of an underactuated AUV, by taking advantages of the time scale separation between the translational dynamics and orientation dynamics. However, no analytical results are provided. With this problem, Lei [27] provides a singular perturbation analysis for the dive control of underactuated AUVs.

In this paper, a method is presented for the path following control of underactuated AUVs subject to model uncertainties and unknown environmental disturbances. To provide an easy-to-implement controller, we resort to the time scald decomposition method and singular perturbation theory. In the present study, the design of output-feedback controller is divided into two parts: the design of ESO and the design of stabilizing controller. The proposed ESO is given in term of the singular perturbation theory. In that case, the asymptotic stability of the proposed ESO does not rely on the 
condition that derivative of the disturbance is zero, as in the earlier study [28]. It is one of the contributions of this paper. The stabilizing controller is devised based on the time scale decomposition method. Specifically, the stabilizing control system is considered as a three time scale singularly perturbed system. Singular perturbation theory is thus applied to analyze the system properties via order reduction. Then a simple stabilizing control law is designed in each lower subsystem independently. And the stability of the stabilizing control system is performed by presenting a stability analysis for generic three-time scale singularly perturbed systems, which allows to construct a composite Lyapunov function for the resultant closed-loop system by using time scale separation. Meanwhile, the mathematical expressions for the upper bounds of the control gains are provided. Finally, its control performance is evaluated through computer simulations. These simulation studies consider two different reference paths. They show that the proposed control scheme is robust in the presence of unknown internal and external disturbances, and a good path following performance is guaranteed.

The remainder of the paper is organized as follows. Section 2 states the problem formulation. Section 3 presents the design and analysis of path following controller via singular perturbation technique and time scale decomposition method. Section 4 presents the simulation results to illustrate the control performance. Section 5 concludes this paper.

\section{Preliminaries}

This section introduces the preliminaries. The inertial frame $\{I\}$ and the body-fixed frame $\{B\}$ are first defined, as depicted in Fig. 1. On the basis of that, the kinematic and kinetic dynamic models of an AUV are presented, and a formulation of the path following control problem of an AUV in the vertical plane is stated.

Table 1. Definition of symbols

\begin{tabular}{c|l}
\hline Symbol & Description \\
\hline$(x, y, z)$ & vehicle's positions with respect to the frame $\{\mathrm{I}\}$ \\
$(u, v, w)$ & vehicle's translational velocities (surge, sway and heave, respectively) expressed in the frame $\{\mathrm{B}\}$ \\
$(\phi, \theta, \psi)$ & vehicle's attitude (the roll, pitch and yaw angles, respectively) \\
$(p, q, r)$ & vehicle's angular velocities about each of the axis of the frame $\{\mathrm{B}\}$ \\
\hline
\end{tabular}

\section{1. model of an AUV in the vertical plane}

The AUV performs underwater missions in a three dimensional plane and has a motion of six degrees of freedom. To reduce the complexity of control design, it is usually decoupled into the vertical motion and the horizontal motion, and considered independently in the controller design [29, 30]. In the present study, we focus on the diving motion of an AUV, and therefore ignore its dynamics of roll, yaw and sway. Accordingly, the motion of an AUV in the vertical plane can be can be expressed as kinematic equations

$$
\left\{\begin{array}{l}
\dot{x}=u \cos \theta+w \sin \theta \\
\dot{z}=-u \sin \theta+w \cos \theta \\
\dot{\theta}=q
\end{array}\right.
$$

and kinetic equations 


$$
\left\{\begin{array}{l}
m_{11} \dot{u}=\varpi_{u}(t, u, w, q)+\tau_{u}+d_{u} \\
m_{33} \dot{w}=\varpi_{w}(t, u, w, q)+\tau_{w}+d_{w} \\
m_{55} \dot{q}=\varpi_{q}(t, u, w, q)+\tau_{q}+d_{q}
\end{array}\right.
$$

where, $m_{11}, m_{33}, m_{55}$ denote mass including hydrodynamic added mass; $\tau_{u}, \tau_{w}$ and $\tau_{q}$ are control inputs; $d_{u}$, $d_{w}$ and $d_{q}$ account for the unknown internal and external disturbances; and $\varpi_{u}(t, u, w, q), \varpi_{w}(t, u, w, q)$ and $\varpi_{q}(t, u, w, q)$ are nonlinear functions related to hydrodynamic damping effects, which are totally unknown.

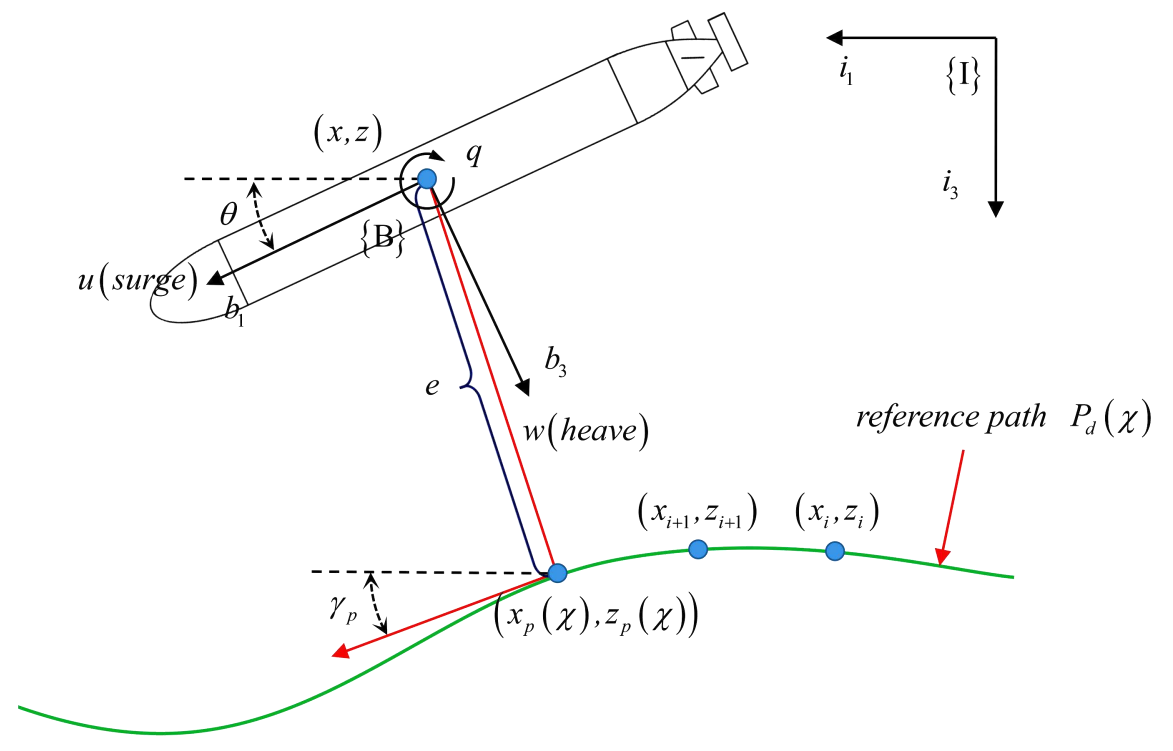

Fig. 1. Vehicle model and reference frames.

\section{2. kinematic path following error dynamics}

In this subsection, the kinematic error dynamics for path following is derived. Let $e$ be the cross-track error. Referring to [31], it is given by

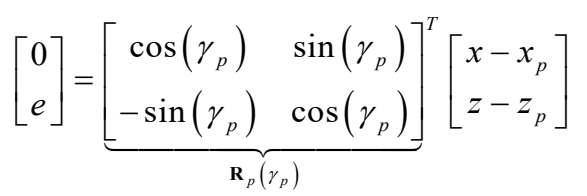

Here, $x_{p}$ and $z_{p}$ are the positions of a point along the reference path; $\gamma_{p}=-\arctan \left(\dot{z}_{p} / \dot{x}_{p}\right)$ denotes the path-tangential angle; and $\mathbf{R}_{p}\left(\gamma_{p}\right) \in S O(2)$ is a rotation matrix.

Moreover, let $\gamma_{e}$ be the path angle tracking error, $u_{e}$ and $w_{e}$ be the velocity tracking errors, $\chi_{e}$ be the pitching rate tracking error. They are expressed as

$$
\left\{\begin{array}{l}
\gamma_{e}=\theta-\alpha-\gamma_{p}, \chi_{e}=q-\dot{\alpha}-\dot{\gamma}_{p} \\
u_{e}=u-u_{d}, w_{e}=w-w_{d}
\end{array}\right.
$$

where $u_{d}$ and $w_{d}$ denote the desired surge and heave velocities, respectively; $\alpha=\operatorname{atan} 2(w, u)$ is the commonly known as the angle of attack (AoA). 
By taking the time derivatives of $e, \gamma_{e}, u_{e}, w_{e}$ and $\chi_{e}$, the obtained error dynamics is as follows:

$$
\left\{\begin{array}{l}
\dot{e}=-U \sin \left(\gamma_{e}\right) \\
\dot{\gamma}_{e}=\chi_{e} \\
\dot{u}_{e}=m_{11}^{-1}\left[\varpi_{u}(t, u, w, q)+\tau_{u}+d_{u}\right]-\dot{u}_{d} \\
\dot{w}_{e}=m_{33}^{-1}\left[\varpi_{w}(t, u, w, q)+\tau_{w}+d_{w}\right]-\dot{w}_{d} \\
\dot{\chi}_{e}=m_{55}^{-1}\left[\varpi_{q}(t, u, w, q)+\tau_{q}+d_{q}\right]-\ddot{\alpha}-\ddot{\gamma}_{p}
\end{array}\right.
$$

Here, $U=\sqrt{u^{2}+w^{2}}$ is referred as the moving speed. The control goal is to design a control law for $\tau_{u}, \tau_{w}$ and $\tau_{q}$ in (5) to stabilize the cross-track error $e$ to zero, so as to force an AUV to follow a prescribed path, with assumption of that the positions and orientation of the vehicle are obtainable.

\section{CONTROL DESIGN AND ANALYSIS}

This section presents the design and analysis of the path following controller, by using the singular perturbation theory and time scale separation.

\section{1 observer design}

In this subsection, a simple observer for the unknown velocities and disturbances is given. To facilitate the observer design, we define

$$
\left\{\begin{array}{l}
\eta=[x, z, \theta]^{T}, \vartheta=[u, w, q]^{T}, \tau=\left[\tau_{u}, \tau_{w}, \tau_{q}\right]^{T} \\
d=\left[d_{u}, d_{w}, d_{q}\right]^{T}, \varpi(t, u, w, q)=\left[\varpi_{u}(\cdot), \varpi_{w}(\cdot), \varpi_{q}(\cdot)\right]^{T} \\
M=\operatorname{diag}\left\{m_{11}, m_{33}, m_{55}\right\}
\end{array}\right.
$$

Then, the control system described by (1) and (2) can be rewritten in a compact form

$$
\left\{\begin{array}{l}
\dot{\eta}=R(\theta) \vartheta \\
\dot{\vartheta}=\sigma+M_{e}^{-1} \tau
\end{array}\right.
$$

here, $R(\theta) \in \mathfrak{R}^{3 \times 3}$ is a rotate matrix defined as

$$
R(\theta)=\left[\begin{array}{ccc}
\cos (\theta) & \sin (\theta) & 0 \\
-\sin (\theta) & \cos (\theta) & 0 \\
0 & 0 & 1
\end{array}\right]
$$

$M_{e}=\operatorname{diag}\left\{m_{e_{-} 11}, m_{e_{-} 33}, m_{e_{-} 55}\right\}$, where $m_{e_{-} 11}, m_{e_{-} 33}$ and $m_{e_{-} 55}$ denote the estimated mass including hydrodynamic added mas. $\sigma=\left[\sigma_{u}, \sigma_{w}, \sigma_{q}\right]^{T}$ accounts for the unknown disturbances and is given by

$$
\sigma=M^{-1}(\varpi(t, u, w, q)+d)+\left(M^{-1}-M_{e}^{-1}\right) \tau
$$

Let $\bar{\eta}=[\bar{x}, \bar{z}, \bar{\theta}]^{T}, \bar{\vartheta}=[\bar{u}, \bar{w}, \bar{q}]^{T}$ and $\bar{\sigma}=\left[\bar{\sigma}_{u}, \bar{\sigma}_{w}, \bar{\sigma}_{q}\right]^{T}$, where $\bar{x}, \bar{z}, \bar{\theta}, \bar{u}, \bar{w}, \bar{q}, \bar{\sigma}_{u}, \bar{\sigma}_{w}$ and $\overline{\sigma_{q}}$ are the estimates of $x, z, \theta, u, w, q, \sigma_{u}, \sigma_{w}$ and $\sigma_{q}$, respectively.

Then we define the state observer as 


$$
\left\{\begin{array}{l}
\dot{\bar{\eta}}=K_{1}(\eta-\bar{\eta})+R(\theta) \bar{\vartheta} \\
\dot{\bar{\vartheta}}=K_{2} R^{T}(\theta)(\eta-\bar{\eta})+\bar{\sigma}+M_{e}^{-1} \tau
\end{array}\right.
$$

To determine the estimate of $\sigma$, we first suppose that $\vartheta$ is known. Then by using the singular perturbation theory [19, 20] and high-gain technique as in [32], the actual disturbance can be computed as

$$
\sigma=K_{3}\left[\vartheta-\int_{0}^{t} M_{e}^{-1} \tau_{1}\left(\eta_{e}, \bar{\vartheta}_{e}\right) d \kappa\right]-\tau_{2}
$$

Here, $\tau_{1}\left(\eta_{e}, \bar{\vartheta}_{e}\right)$ is the so called stabilizing controller, which satisfies $\tau=\tau_{1}\left(\eta_{e}, \bar{\vartheta}_{e}\right)-M_{e} \bar{\sigma} . \tau_{2}$ is given by

$$
\dot{\tau}_{2}=-K_{3} M_{e}^{-1} \tau_{2}
$$

So the estimate of $\sigma$ can be taken as

$$
\bar{\sigma}=K_{3}\left[\bar{\vartheta}-\int_{0}^{t} M_{e}^{-1} \tau_{1}\left(\eta_{e}, \bar{\vartheta}_{e}\right) d \kappa\right]
$$

In (10) and (11), $K_{1}=\operatorname{diag}\left\{k_{11}, k_{12}, k_{13}\right\}, K_{2}=\operatorname{diag}\left\{k_{21}, k_{22}, k_{23}\right\} \quad$ and $K_{3}=\operatorname{diag}\left\{k_{31}, k_{32}, k_{33}\right\} \quad$ are constant matrices, where $k_{(\cdot)}$ are positive constants.

Let errors be

$$
\left\{\begin{array}{l}
\widehat{\eta}=\eta-\bar{\eta} \\
\widehat{\vartheta}=\vartheta-\bar{\vartheta}
\end{array}\right.
$$

The error dynamics can be taken as

$$
\left\{\begin{array}{l}
\dot{\bar{\eta}}=-K_{1} \hat{\eta}+R(\theta) \hat{\vartheta} \\
\dot{\bar{\vartheta}}=-K_{2} R^{T}(\theta) \hat{\eta}+\left(K_{3} \widehat{\vartheta}-\tau_{2}\right) \\
\dot{\tau}_{2}=-K_{3} M_{e}^{-1} \tau_{2}
\end{array}\right.
$$

Let $Y=\left[\widehat{\eta}^{T}, \widehat{\vartheta}^{T}, \tau_{2}^{T}\right]^{T}$, system (15) is put into

$$
\dot{Y}=-A Y
$$

where

$$
A=\left[\begin{array}{ccc}
K_{1} & -R(\theta) & 0_{3} \\
K_{2} R^{T}(\theta) & -K_{3} & I_{3} \\
0_{3} & 0_{3} & K_{3} M_{e}^{-1}
\end{array}\right]
$$

It is clear to see that the system is globally uniformly asymptotically stable, in case of $A$ is positive definite.

Remark: Compared with the ESO as in [28], the proposed ESO has an obvious feature that the stability of system (15) does not require condition $\dot{\sigma}=0$.

\subsection{Stabilizing controller}

In this subsection, the design of stabilizing controller is presented, in term of the time scale decomposition method.

To facilitate the control design, we apply the separation principle. Then our control goal herein is to design a control for $\tau_{1}\left(\eta_{e}, \vartheta_{e}\right)=\left[\tau_{1_{-} u}, \tau_{1_{-} w}, \tau_{1_{-} q}\right]^{T}$ in the following system (18) to stabilize the cross-track error $e$ to zero. 


$$
\left\{\begin{array}{l}
\dot{e}=-U \sin \left(\gamma_{e}\right) \\
\dot{\gamma}_{e}=\chi_{e} \\
\dot{u}_{e}=m_{e_{-} 11}^{-1} \tau_{1_{1} u}-\dot{u}_{d} \\
\dot{w}_{e}=m_{e_{-} 33}^{-1} \tau_{1_{-} w}-\dot{w}_{d} \\
\dot{\chi}_{e}=m_{e_{-} 55}^{-1} \tau_{1_{-} q}-\ddot{\alpha}-\ddot{\gamma}_{p}
\end{array}\right.
$$

On an initial inspection of model (18), one might find that the backstepping control technique might be an effective method. However, it yields a relatively complicated controller. To obtain a simpler control law, which should be easy-to-implement in practical cases, the time scale decomposition method is applied.

It is well established that singular perturbation and hence the time scale (slow-fast) character is often associated with a small parameter multiplying the highest derivative of the differential equation or multiplying some of the state variables of the state equation describing a physical system. However, modeling a physical system in the singularly perturbed form may not be easy, due to the fact that it is not always clear how to identify the small singularly perturbed parameters [33]. Following the approach as in [34,35], in this section, a forced singular perturbation model is obtained, by artificial insertion of the singularly perturbed parameters. We select $e$ as the slow variable; $\gamma_{e}$ as fast variable; $u_{e}, w_{e}$ and $\chi_{e}$ as the ultra-fast variables. Then, system (18) can be taken as the following three time scale singularly perturbed form:

$$
\left\{\begin{array}{l}
\dot{e}=-U \sin \left(\gamma_{e}\right) \\
\varepsilon_{1} \dot{\gamma}_{e}=\mu_{1} \chi_{e} \\
\varepsilon_{1} \varepsilon_{2} \dot{u}_{e}=\mu_{2}\left[m_{e_{-} 11}^{-1} \tau_{1_{-u}}-\dot{u}_{d}\right] \\
\varepsilon_{1} \varepsilon_{2} \dot{w}_{e}=\mu_{2}\left[m_{e_{-} 33}^{-1} \tau_{1_{-w} w}-\dot{w}_{d}\right] \\
\varepsilon_{1} \varepsilon_{2} \dot{\chi}_{e}=\mu_{2}\left[m_{e_{-} 55}^{-1} \tau_{1_{-} q}-\ddot{\alpha}-\ddot{\gamma}_{p}\right]
\end{array}\right.
$$

Here, $\varepsilon_{i=1,2}$ are artificial small positive constants in order to provide a singular perturbation analysis; $\mu_{1}=\varepsilon_{1}$; $\mu_{2}=\varepsilon_{1} \varepsilon_{2}$.

\subsection{1 time scale decomposition}

This part presents the time scale decomposition of system (19). For convenience, we rewrite the three-time scale singularly perturbed system (19) in a compact form as follows:

$$
\left\{\begin{array}{l}
\dot{\boldsymbol{x}}=f(\boldsymbol{x}, \boldsymbol{y}, \boldsymbol{z}) \\
\varepsilon_{1} \dot{\boldsymbol{y}}=g(\boldsymbol{x}, \boldsymbol{y}, \boldsymbol{z}) \\
\varepsilon_{1} \varepsilon_{2} \dot{\boldsymbol{z}}=h(\boldsymbol{x}, \boldsymbol{y}, \boldsymbol{z})
\end{array}\right.
$$

Here, $\boldsymbol{x}=e, \quad \boldsymbol{y}=\gamma_{e}$ and $\boldsymbol{z}=\left[u_{e}, w_{e}, \chi_{e}\right]^{T}$ denote slow, fast and ultra-fast state variables of the three-time scale singularly perturbed system (19), respectively. $f(\cdot), g(\cdot)$ and $h(\cdot)$ are continuously differentiable functions of state variables $\boldsymbol{x}, \boldsymbol{y}$ and $\boldsymbol{z}$.

Prior to time scale decomposition, we define operators ${ }^{\wedge}$ and $\sim$, which yield the quasi-steady-state and the boundary layer correction of a state variable. For more detail about these definitions, one might refer to [19,20]. On the basis of that, an intuitive description of singular perturbation reduction can be seen in Fig. 2. Specifically, the generic three-time scale system (20) is sequentially decomposed into two distinct two-time scale models. The first two-time scale model, 
that is, Layer-1, is obtained by considering the stretched time scale $t_{2}=t_{1} / \varepsilon_{2} \triangleq t /\left(\varepsilon_{1} \varepsilon_{2}\right)$, where the Layer-1 fast subsystem, is defined by

$$
\frac{d \tilde{z}}{d t_{2}}=h(\boldsymbol{x}, \boldsymbol{y}, \tilde{\boldsymbol{z}}+H(\boldsymbol{x}, \boldsymbol{y}))
$$

and the Layer-1 slow subsystem, is defined by

$$
\left\{\begin{array}{r}
\dot{\boldsymbol{x}}=f(\boldsymbol{x}, \boldsymbol{y}, H(\boldsymbol{x}, \boldsymbol{y})) \\
\varepsilon_{1} \dot{\boldsymbol{y}}=g(\boldsymbol{x}, \boldsymbol{y}, H(\boldsymbol{x}, \boldsymbol{y}))
\end{array}\right.
$$

Note that, $\boldsymbol{x}$ and $\boldsymbol{y}$ are treated as frozen variables during the fast transient (21). $H(\boldsymbol{x}, \boldsymbol{y})$ represents the quasi-steady-state of $\boldsymbol{z}$, given by setting $\varepsilon_{2}=0$, that is, $0=h(\boldsymbol{x}, \boldsymbol{y}, \boldsymbol{z}) \rightarrow \hat{\boldsymbol{z}}=H(\boldsymbol{x}, \boldsymbol{y})$. Similarly, the reduced order Layer-1 slow subsystem (22) can be treated again like a two-time scale singular perturbation problem by considering the stretched time scale $t_{1}=t / \varepsilon_{1}$, where the boundary-layer subsystem, denoted by the Layer-2 fast subsystem, is defined by

$$
\frac{d \tilde{\boldsymbol{y}}}{d t_{1}}=g(\boldsymbol{x}, \tilde{\boldsymbol{y}}+G(\boldsymbol{x}), H(\boldsymbol{x}, \tilde{\boldsymbol{y}}+G(\boldsymbol{x})))
$$

and where the reduced subsystem, denoted by the Layer-2 slow subsystem, is defined by

$$
\dot{\boldsymbol{x}}=f(\boldsymbol{x}, G(\boldsymbol{x}), H(\boldsymbol{x}, G(\boldsymbol{x})))
$$

Also note, $\boldsymbol{x}$ is treated as a frozen variable during the fast transient (23). $G(\boldsymbol{x})$ denotes the quasi-steady-state of $\boldsymbol{y}$, obtained by setting $\varepsilon_{1}=0$, that is, $0=g(\boldsymbol{x}, \boldsymbol{y}, H(\boldsymbol{x}, \boldsymbol{y})) \rightarrow \hat{\boldsymbol{y}}=G(\boldsymbol{x})$, with assumption of that $\boldsymbol{z}$ has reached its quasi-steady-state and evolve on its own manifold.

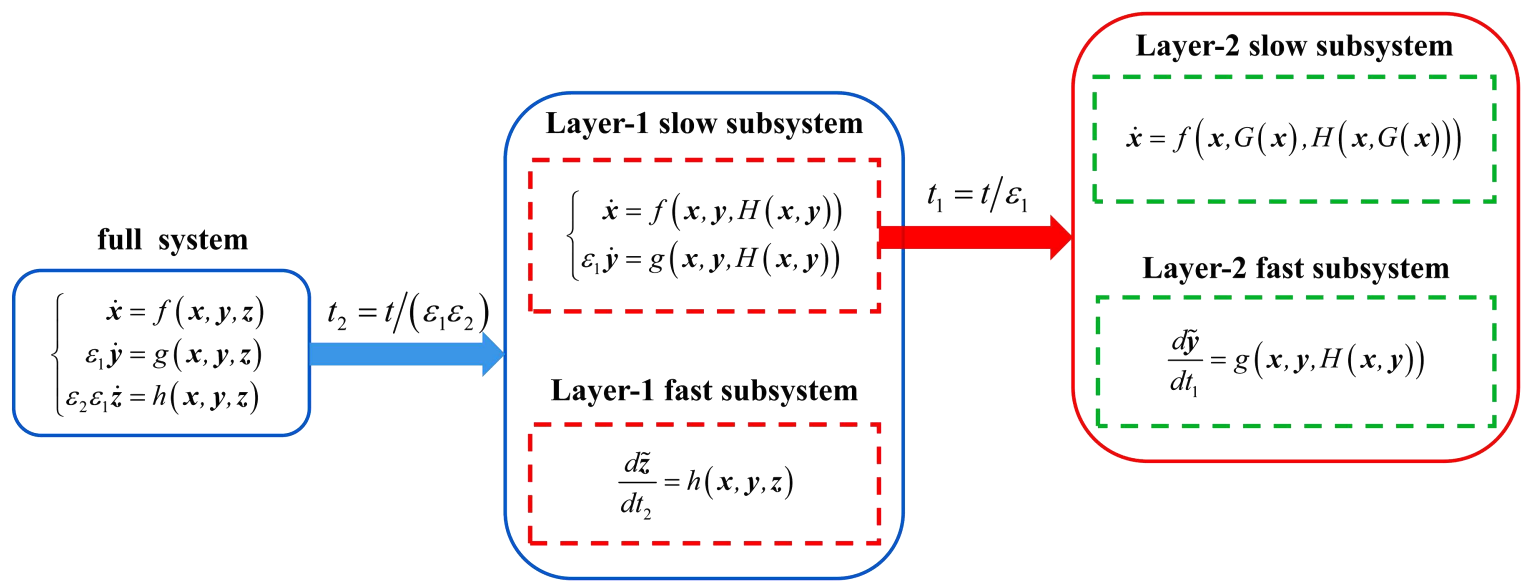

Fig. 2. Three-time scale singular perturbation reduction

\subsection{2 control design}

In this part, the control laws will be designed in each subsystem separately, in term of the singular perturbation theory.

We start with the Layer-2 slow subsystem. It can be seen that the Layer-2 slow subsystem (24) is asymptotically stable by selecting a virtual feedback control law

$$
\hat{\gamma}_{e}=k_{1} e
$$

Here, $k_{1}>0$. Note that, the design parameter $k_{1}$ should satisfy condition $\left|k_{1} e\right| \leq \pi / 2$. 
Proof. Consider a Lyapunov function candidate

$$
V_{2}(\boldsymbol{x})=\frac{1}{2} e^{2}
$$

Derivative of $V_{2}(\boldsymbol{x})$ along the trajectories of system (24) can be taken as

$$
\dot{V}_{2}(\boldsymbol{x})=\frac{\partial V_{2}(\boldsymbol{x})}{\partial \boldsymbol{x}} f(\boldsymbol{x}, G(\boldsymbol{x}), H(\boldsymbol{x}, G(\boldsymbol{x})))=-e \hat{U} \sin \left(k_{1} e\right)
$$

In this present study, we set $u_{d}=1 \mathrm{~m} / \mathrm{s}$ and $w_{d}=0 \mathrm{~m} / \mathrm{s}$, therefore $\hat{U}=1$. Since $k_{1}>0,\left|k_{1} e\right| \leq \pi / 2$ and $U>0$, we compute $\dot{V}_{2}(\boldsymbol{x}) \leq 0$ and $\dot{V}_{2}(\boldsymbol{x})<0, \forall \boldsymbol{x} \neq 0$. Thereby, the asymptotic stability of system (24) near the equilibrium point is guaranteed.

Following that, the Layer-2 fast subsystem (23) is asymptotically stable by selecting a virtual feedback control law

$$
\hat{\chi}_{e}=k_{2}\left(k_{1} e-\gamma_{e}\right)
$$

where, $k_{2}>0$.

Proof. Consider a Lyapunov function candidate

$$
W_{2}(\boldsymbol{x}, \tilde{\boldsymbol{y}})=\frac{1}{2} \tilde{\gamma}_{e}^{2}
$$

We have

$$
\dot{W}_{2}(\boldsymbol{x}, \tilde{\boldsymbol{y}})=\frac{\partial W_{2}(\boldsymbol{x}, \tilde{\boldsymbol{y}})}{\partial \tilde{\boldsymbol{y}}} \frac{1}{\mu_{1}} g(\boldsymbol{x}, \tilde{\boldsymbol{y}}+G(\boldsymbol{x}), H(\boldsymbol{x}, \tilde{\boldsymbol{y}}+G(\boldsymbol{x})))=-k_{2} \tilde{\gamma}_{e}^{2}
$$

As $k_{2}>0$, it is clear to see that $\dot{W}_{2}(\boldsymbol{x}, \tilde{\boldsymbol{y}}) \leq 0$ and $\dot{W}_{2}(\boldsymbol{x}, \tilde{\boldsymbol{y}})<0, \forall \tilde{\boldsymbol{y}} \neq 0$. Therefore, system (23) is asymptotically stable.

Finally, the Layer-1 fast subsystem (21) will be asymptotically stable by selecting

$$
\left\{\begin{array}{l}
\tau_{1_{-} u}=m_{e_{-} 11}\left(-k_{3} u_{e}+\dot{u}_{d}\right) \\
\tau_{1_{-} w}=m_{e_{-} 33}\left(-k_{4} w_{e}+\dot{w}_{d}\right) \\
\tau_{1_{-} q}=m_{e_{-} 55}\left(-k_{5} \tilde{\chi}_{e}+\ddot{\alpha}+\ddot{\gamma}_{p}\right)
\end{array}\right.
$$

where, $k_{i=3,4,5}>0$.

Proof. Consider a Lyapunov function candidate

$$
W_{1}=\left(\tilde{u}_{e}^{2}+\tilde{w}_{e}^{2}+\tilde{\chi}_{e}^{2}\right) / 2
$$

We compute

$$
\dot{W}_{1}(\boldsymbol{x}, \boldsymbol{y}, \tilde{\boldsymbol{z}})=\frac{\partial W_{1}(\boldsymbol{x}, \boldsymbol{y}, \tilde{\boldsymbol{z}})}{\partial \tilde{\boldsymbol{z}}} \frac{1}{\mu_{2}} h(\boldsymbol{x}, \boldsymbol{y}, \tilde{\boldsymbol{z}}+H(\boldsymbol{x}, \boldsymbol{y}))=-k_{3} \tilde{u}_{e}^{2}-k_{4} \tilde{w}_{e}^{2}-k_{5} \tilde{\chi}_{e}^{2}
$$

As $k_{i=3,4,5}>0$, it is clear to see that $\dot{W}_{1}(\boldsymbol{x}, \boldsymbol{y}, \tilde{\boldsymbol{z}}) \leq 0$ and $\dot{W}_{1}(\boldsymbol{x}, \boldsymbol{y}, \tilde{\boldsymbol{z}})<0, \forall \tilde{\boldsymbol{z}} \neq 0$. So, considering the control input as in (31), the origin of the Layer-1 fast subsystem (21) is an asymptotically stable equilibrium point.

Remark: Substituting the estimated velocities into (31) will yield the actual stabilizing controller. As illustration above, the proposed control law can be designed independently and thus being easily obtained, leading to a reduction of control 
complexity. And it is clear to see that, compared with the traditional backstepping control technique, the proposed controller is relatively simple and easy-to-implement.

\subsection{3 stability analysis}

Stability of the lower order subsystems guarantees only the local stability of the equilibrium of the full dynamics (19). In this subsection, we will construct a composite Lyapunov function candidate for full dynamics (19), and provide mathematical expressions for the upper bounds of those control gains.

Prior to that, we rewrite system (20) as follow:

$$
\left\{\begin{array}{l}
\dot{\boldsymbol{x}}=f(\boldsymbol{x}, G(\boldsymbol{x}), H(\boldsymbol{x}, G(\boldsymbol{x})))+[f(\boldsymbol{x}, \boldsymbol{y}, H(\boldsymbol{x}, \boldsymbol{y}))-f(\boldsymbol{x}, G(\boldsymbol{x}), H(\boldsymbol{x}, G(\boldsymbol{x})))]+\Delta F \\
\dot{\tilde{\boldsymbol{y}}}=\frac{1}{\varepsilon_{1}} g(\boldsymbol{x}, \boldsymbol{y}, H(\boldsymbol{x}, \boldsymbol{y}))-\frac{\partial G(\boldsymbol{x})}{\partial \boldsymbol{x}} \dot{\boldsymbol{x}}+\Delta G \\
\dot{\tilde{\boldsymbol{z}}}=\frac{1}{\varepsilon_{1} \varepsilon_{2}} h(\boldsymbol{x}, \boldsymbol{y}, \boldsymbol{z})-\frac{\partial H(\boldsymbol{x}, \boldsymbol{y})}{\partial(\boldsymbol{x}, \boldsymbol{y})}\left[\begin{array}{l}
\dot{\boldsymbol{x}} \\
\dot{\boldsymbol{y}}
\end{array}\right]
\end{array}\right.
$$

Here,

$$
\begin{gathered}
\Delta F=f(\boldsymbol{x}, \boldsymbol{y}, \tilde{z}+H(\boldsymbol{x}, \boldsymbol{y}))-f(\boldsymbol{x}, \boldsymbol{y}, H(\boldsymbol{x}, \boldsymbol{y})) \\
\Delta G=\frac{1}{\varepsilon_{1}}[g(\boldsymbol{x}, \boldsymbol{y}, \tilde{z}+H(\boldsymbol{x}, \boldsymbol{y}))-g(\boldsymbol{x}, \boldsymbol{y}, H(\boldsymbol{x}, \boldsymbol{y}))]
\end{gathered}
$$

Proposition 4. Referring to [36-38], there is a composite Lyapunov function candidate

$$
V(\boldsymbol{x}, \tilde{\boldsymbol{y}}, \tilde{z})=\left(1-n_{1}\right)\left[\left(1-n_{2}\right) V_{2}(\boldsymbol{x})+n_{2} W_{2}(\boldsymbol{x}, \tilde{\boldsymbol{y}})\right]+n_{1} W_{1}(\boldsymbol{x}, \boldsymbol{y}, \tilde{z})
$$

for proving the asymptotic stability of the the origin of three time scale singularly perturbed system (20). Here, $0<n_{i=1,2}<1$.

Proof. The derivative $\dot{V}(\boldsymbol{x}, \tilde{\boldsymbol{y}}, \tilde{\boldsymbol{z}})$ is given by

$$
\begin{aligned}
\dot{V}(\boldsymbol{x}, \tilde{\boldsymbol{y}}, \tilde{\boldsymbol{z}})= & \left(1-n_{1}\right)\left(1-n_{2}\right) \frac{\partial V_{2}(\boldsymbol{x})}{\partial \boldsymbol{x}} f(\boldsymbol{x}, G(\boldsymbol{x}), H(\boldsymbol{x}, G(\boldsymbol{x})))+\left(1-n_{1}\right) \frac{n_{2}}{\mu_{1}} \frac{\partial W_{2}(\boldsymbol{x}, \tilde{\boldsymbol{y}})}{\partial \tilde{\boldsymbol{y}}} g(\boldsymbol{x}, \boldsymbol{y}, H(\boldsymbol{x}, \boldsymbol{y})) \\
& +\left(1-n_{1}\right)\left(1-n_{2}\right) \frac{\partial V_{2}(\boldsymbol{x})}{\partial \boldsymbol{x}}[f(\boldsymbol{x}, \boldsymbol{y}, H(\boldsymbol{x}, \boldsymbol{y}))-f(\boldsymbol{x}, G(\boldsymbol{x}), H(\boldsymbol{x}, G(\boldsymbol{x})))] \\
& +\left(1-n_{1}\right) n_{2}\left[\frac{\partial W_{2}(\boldsymbol{x}, \tilde{\boldsymbol{y}})}{\partial \boldsymbol{x}}-\frac{\partial W_{2}(\boldsymbol{x}, \tilde{\boldsymbol{y}})}{\partial \tilde{\boldsymbol{y}}} \frac{\partial G(\boldsymbol{x})}{\partial \boldsymbol{x}}\right] f(\boldsymbol{x}, \boldsymbol{y}, H(\boldsymbol{x}, \boldsymbol{y})) \\
& +\left(1-n_{1}\right)\left\{\frac{\partial\left[\left(1-n_{2}\right) V_{2}(\boldsymbol{x})+n_{2} W_{2}(\boldsymbol{x}, \tilde{\boldsymbol{y}})\right]}{\partial(\boldsymbol{x}, \tilde{\boldsymbol{y}})}\left[\begin{array}{c}
\Delta F \\
\Delta G
\end{array}\right]\right\} \\
& +n_{1}\left\{\frac{\partial W_{1}(\boldsymbol{x}, \boldsymbol{y}, \tilde{\boldsymbol{z}})}{\partial(\boldsymbol{x}, \boldsymbol{y})}-\frac{\partial W_{1}(\boldsymbol{x}, \boldsymbol{y}, \tilde{\boldsymbol{z}})}{\partial \tilde{\boldsymbol{z}}} \frac{\partial H(\boldsymbol{x}, \boldsymbol{y})}{\partial(\boldsymbol{x}, \boldsymbol{y})}\right\}\left[\begin{array}{c}
\dot{\boldsymbol{x}} \\
\dot{\boldsymbol{y}}
\end{array}\right]+\frac{n_{1}}{\mu_{2}} \frac{\partial W_{1}(\boldsymbol{x}, \boldsymbol{y}, \tilde{\boldsymbol{z}})}{\partial \tilde{\boldsymbol{z}}} h(\boldsymbol{x}, \boldsymbol{y}, \boldsymbol{z})
\end{aligned}
$$

Let the following conditions hold:

(1) $\frac{\partial V_{2}(\boldsymbol{x})}{\partial \boldsymbol{x}} f(\boldsymbol{x}, G(\boldsymbol{x}), H(\boldsymbol{x}, G(\boldsymbol{x}))) \leq-\alpha_{1} \varphi_{1}^{2}(\boldsymbol{x})$; 
(2) $\frac{\partial W_{2}(\boldsymbol{x}, \tilde{\boldsymbol{y}})}{\partial \tilde{\boldsymbol{y}}} \frac{1}{\mu_{1}} g(\boldsymbol{x}, \boldsymbol{y}, H(\boldsymbol{x}, \boldsymbol{y})) \leq-\alpha_{2} \varphi_{2}^{2}(\tilde{\boldsymbol{y}})$;

(3) $\frac{\partial V_{2}(\boldsymbol{x})}{\partial \boldsymbol{x}}[f(\boldsymbol{x}, \boldsymbol{y}, H(\boldsymbol{x}, \boldsymbol{y}))-f(\boldsymbol{x}, G(\boldsymbol{x}), H(\boldsymbol{x}, G(\boldsymbol{x})))] \leq \beta_{1} \varphi_{1}(\boldsymbol{x}) \varphi_{2}(\tilde{\boldsymbol{y}})$;

(4) $\left[\frac{\partial W_{2}(\boldsymbol{x}, \tilde{\boldsymbol{y}})}{\partial \boldsymbol{x}}-\frac{\partial W_{2}(\boldsymbol{x}, \tilde{\boldsymbol{y}})}{\partial \tilde{\boldsymbol{y}}} \frac{\partial G(\boldsymbol{x})}{\partial \boldsymbol{x}}\right] f(\boldsymbol{x}, \boldsymbol{y}, H(\boldsymbol{x}, \boldsymbol{y})) \leq \beta_{2} \varphi_{1}(\boldsymbol{x}) \varphi_{2}(\tilde{\boldsymbol{y}})+\beta_{3} \varphi_{2}^{2}(\tilde{\boldsymbol{y}})$;

(5) $\frac{\partial W_{1}(\boldsymbol{x}, \boldsymbol{y}, \tilde{z})}{\partial \tilde{z}} \frac{1}{\mu_{2}} h(\boldsymbol{x}, \boldsymbol{y}, \boldsymbol{z}) \leq-a_{2} \varphi_{4}^{2}(\tilde{\boldsymbol{z}})$;

(6) $\frac{\partial\left[\left(1-n_{2}\right) V_{2}(\boldsymbol{x})+n_{2} W_{2}(\boldsymbol{x}, \tilde{\boldsymbol{y}})\right]}{\partial(\boldsymbol{x}, \tilde{\boldsymbol{y}})}\left[\begin{array}{c}\Delta F \\ \Delta G\end{array}\right] \leq b_{1} \varphi_{3}(\boldsymbol{x}, \tilde{\boldsymbol{y}}) \varphi_{4}(\tilde{\boldsymbol{z}})$;

(7) $\left[\frac{\partial W_{1}(\boldsymbol{x}, \boldsymbol{y}, \tilde{\boldsymbol{z}})}{\partial(\boldsymbol{x}, \boldsymbol{y})}-\frac{\partial W_{1}(\boldsymbol{x}, \boldsymbol{y}, \tilde{z})}{\partial \tilde{z}} \frac{\partial H(\boldsymbol{x}, \boldsymbol{y})}{\partial(\boldsymbol{x}, \boldsymbol{y})}\right]\left[\begin{array}{c}\dot{\boldsymbol{x}} \\ \dot{\boldsymbol{y}}\end{array}\right] \leq b_{2} \varphi_{3}(\boldsymbol{x}, \tilde{\boldsymbol{y}}) \varphi_{4}(\tilde{z})+b_{3} \varphi_{4}^{2}(\tilde{\boldsymbol{z}})$.

Here, $\varphi_{i=1,2,4}(\cdot)$ are continuous scalar positive-definite functions; $\varphi_{3}(\boldsymbol{x}, \tilde{\boldsymbol{y}})=\left[\varphi_{1}(\boldsymbol{x}), \varphi_{2}(\tilde{\boldsymbol{y}})\right]^{T} ; \quad \alpha_{i=1,2}$ and $a_{2}$ are positive constants; $\beta_{i=1,2,3}$ and $b_{i=1,2,3}$ are nonnegative constants or matrices with nonnegative elements. The derivation of these coefficients and functions is outlined in Appendix.

Substituting assumptions (1) - (7) into (36) yields an inequality

$$
\begin{aligned}
\dot{V}(\boldsymbol{x}, \tilde{\boldsymbol{y}}, \tilde{\boldsymbol{z}})= & \left(1-n_{1}\right)\left(1-n_{2}\right)\left[-\alpha_{1} \varphi_{1}^{2}(\boldsymbol{x})+\beta_{1} \varphi_{1}(\boldsymbol{x}) \varphi_{2}(\tilde{\boldsymbol{y}})\right] \\
& +\left(1-n_{1}\right) n_{2}\left[-\alpha_{2} \varphi_{2}^{2}(\tilde{\boldsymbol{y}})+\beta_{2} \varphi_{1}(\boldsymbol{x}) \varphi_{2}(\tilde{\boldsymbol{y}})+\beta_{2} \varphi_{2}^{2}(\tilde{\boldsymbol{y}})\right] \\
& +\left(1-n_{1}\right)\left\{b_{1} \varphi_{3}(\boldsymbol{x}, \tilde{\boldsymbol{y}}) \varphi_{4}(\tilde{\boldsymbol{z}})\right\} \\
& +n_{1}\left\{b_{2} \varphi_{3}(\boldsymbol{x}, \tilde{\boldsymbol{y}}) \varphi_{4}(\tilde{\boldsymbol{z}})+b_{3} \varphi_{4}^{2}(\tilde{\boldsymbol{z}})\right\}-n_{1} a_{2} \varphi_{4}^{2}(\tilde{\boldsymbol{z}}) \\
\leq & \left(1-n_{1}\right)\left\{-\varphi_{3}^{T}(\boldsymbol{x}, \tilde{\boldsymbol{y}}) \Lambda \varphi_{3}(\boldsymbol{x}, \tilde{\boldsymbol{y}})+b_{1} \varphi_{3}(\boldsymbol{x}, \tilde{\boldsymbol{y}}) \varphi_{4}(\tilde{\boldsymbol{z}})\right\} \\
& +n_{1}\left\{-a_{2} \varphi_{4}^{2}(\tilde{\boldsymbol{z}})+b_{2} \varphi_{3}(\boldsymbol{x}, \tilde{\boldsymbol{y}}) \varphi_{4}(\tilde{z})+b_{3} \varphi_{4}^{2}(\tilde{\boldsymbol{z}})\right\} \\
\leq & -\Psi^{T}(\boldsymbol{x}, \tilde{\boldsymbol{y}}, \tilde{\boldsymbol{z}}) \Pi \Psi(\boldsymbol{x}, \tilde{\boldsymbol{y}}, \tilde{\boldsymbol{z}})
\end{aligned}
$$

where

$$
\Psi(\boldsymbol{x}, \tilde{\boldsymbol{y}}, \tilde{z})=\left[\begin{array}{c}
\varphi_{3}(\boldsymbol{x}, \tilde{\boldsymbol{y}}) \\
\varphi_{4}(\tilde{z})
\end{array}\right] \triangleq\left[\begin{array}{c}
\varphi_{1}(\boldsymbol{x}) \\
\varphi_{2}(\tilde{\boldsymbol{y}}) \\
\varphi_{4}(\tilde{z})
\end{array}\right]
$$

and

$$
\begin{gathered}
\Lambda=\left[\begin{array}{cc}
\left(1-n_{2}\right) \alpha_{1} & -0.5\left[\left(1-n_{2}\right) \beta_{1}+n_{2} \beta_{2}\right] \\
-0.5\left[\left(1-n_{2}\right) \beta_{1}+n_{2} \beta_{2}\right] & n_{2}\left(\alpha_{2}-\beta_{3}\right)
\end{array}\right] \\
\Pi=\left[\begin{array}{cc}
\left(1-n_{1}\right) \Lambda & -0.5\left[\left(1-n_{1}\right) b_{1}+n_{1} b_{2}\right] \\
-0.5\left[\left(1-n_{1}\right) b_{1}+n_{1} b_{2}\right]^{T} & n_{1}\left(a_{2}-b_{3}\right)
\end{array}\right]
\end{gathered}
$$

It is clear to see that the derivative of $V(\boldsymbol{x}, \tilde{\boldsymbol{y}}, \tilde{\boldsymbol{z}})$ along the trajectories of system (20) is negative definite, in case of $\Pi$ is positive definite, namely, the following conditions hold: 


$$
\begin{aligned}
& |\Lambda|_{d}>0 \\
& |\Pi|_{d}>0
\end{aligned}
$$

Here, the operator ||$_{d}$ yields the determinant of a matrix. The detailed calculation is omitted for the sake of brevity. Therefore, the origin of the three-time scale singularly perturbed system (20) is an asymptotically stable equilibrium point for control gains that satisfy the above conditions (38) and (39).

Remark: The motivation to this stability analysis comes from the work conducted by Esteban et al. [36] that used a three time scale singularly perturbation control law for the stabilization of a nonlinear highly coupled helicopter model in vertical flight. However, the methods for the derivation of those coefficients in assumptions (1) - (7) are different. Specifically, the proposed method allows the existence of constant matrices to make $\Pi \in \mathfrak{R}^{n \times n}$, where $n$ denotes the time scales. In that case, the proposed stability analysis can be extended to arbitrary multi-time scale singularly perturbed system. And it must be emphasized that, compared with the method as in [36], the proposed method can obtain larger upper bounds of the design parameters, by reducing the application of Young's inequality.

(a)

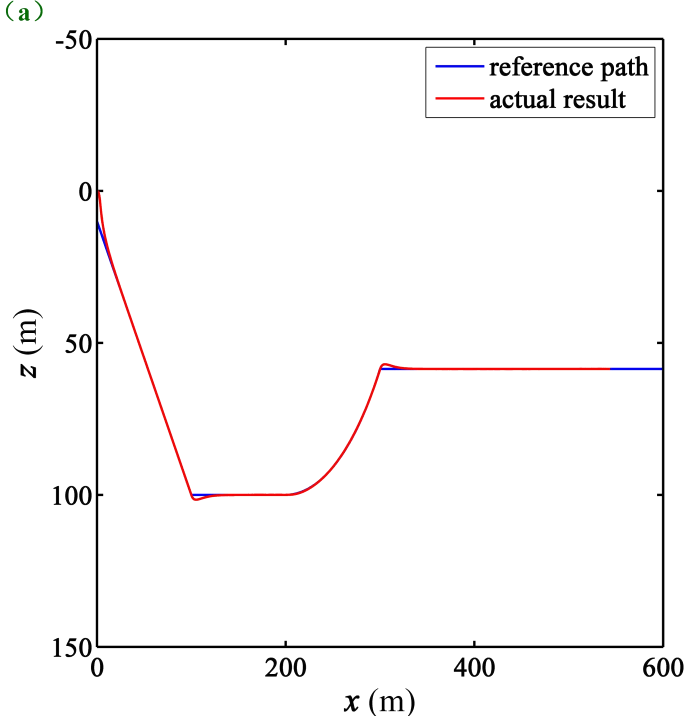

(b)
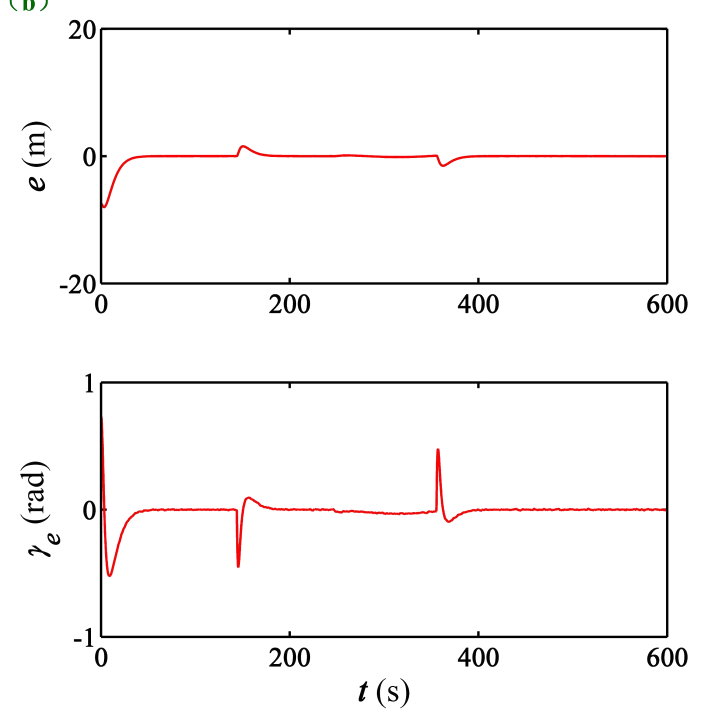

Fig. 3. Simulation results of following a composite path. (a) a composite path (blue solid line: reference path; red solid line: the actual result); (b) cross-track error and path angle tracking error. 
(a)
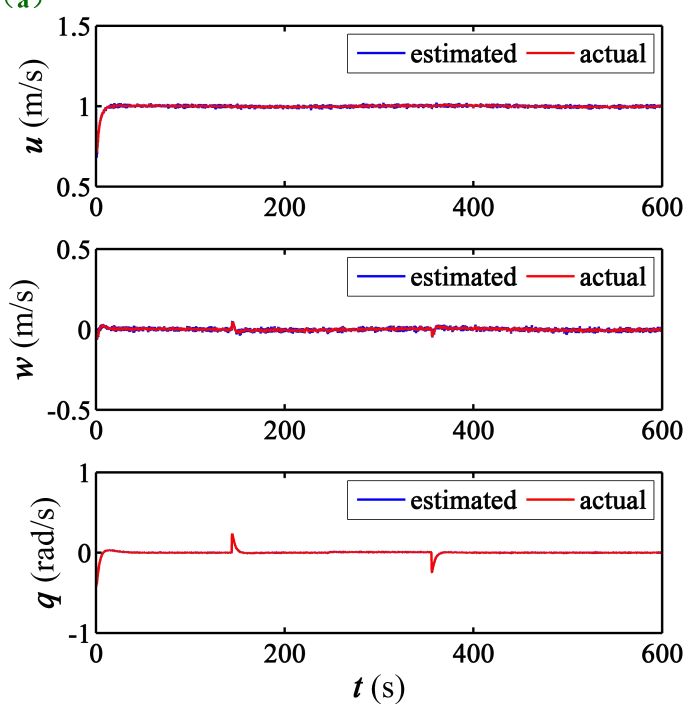

(b)
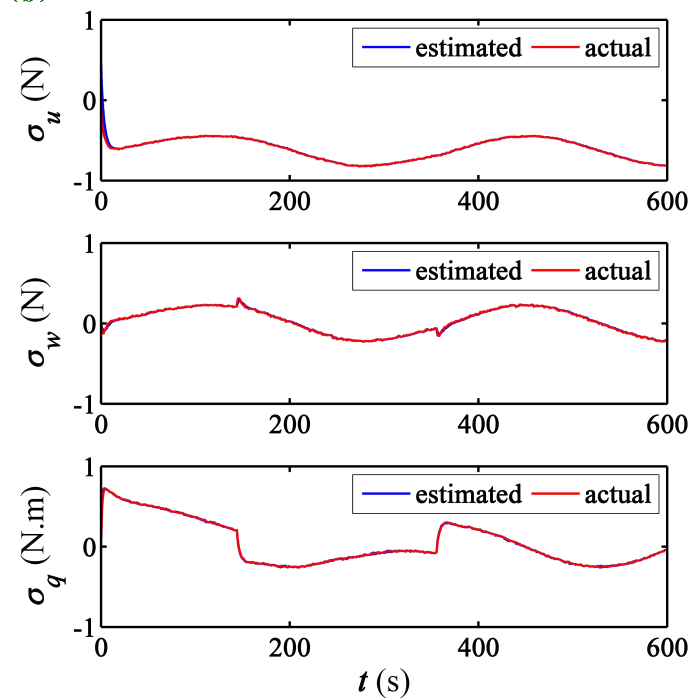

Fig. 4. Estimation performance by using the ESO.

\section{Simulation Results}

In this section, the simulations results will be provided to illustrate the control performance of this closed-loop system, using an AUV model used in earlier studies [9, 39].

$$
\left\{\begin{array}{l}
\dot{u}=\frac{1}{m_{11}}\left\{-m_{33} w q-d_{11} u+\tau_{u}+d_{u}\right\} \\
\dot{w}=\frac{1}{m_{33}}\left\{m_{11} u q-d_{33} w+\tau_{w}+d_{w}\right\} \\
\dot{q}=\frac{1}{m_{55}}\left\{\left(m_{33}-m_{11}\right) u w-d_{55} q-m g d \sin (\theta)+\tau_{q}+d_{q}\right\}
\end{array}\right.
$$

Here, $\quad m_{11}=215, \quad m_{33}=265, \quad m_{55}=80, \quad d_{11}=70+100|u|, \quad d_{33}=100+200|w|, \quad d_{55}=50+100|q|, \quad m=185$, $g=9.8, d=0.02 . d_{u}, d_{w}$ and $d_{q}$ are given by

$$
\left\{\begin{array}{l}
d_{u}=-0.2 \times\left(-m_{33} w q-d_{11} u\right)+40 \times \sin (0.02 t)+40 \times \operatorname{rand}(1) \\
d_{w}=-0.2 \times\left(m_{11} u q-d_{33} w\right)+60 \times \sin (0.02 t)+60 \times \operatorname{rand}(1) \\
d_{q}=-0.2 \times\left[\left(m_{33}-m_{11}\right) u w-d_{55} q\right]+20 \times \cos (0.02 t)+10 \times \text { rand }(1)
\end{array}\right.
$$

where, rand(1) is the zero-mean random noise with amplitude of 1 . The three terms on the right hand side of (41) account for the errors of the order of $20 \%$ on system parameters, periodic unknown disturbances and random noise, respectively.

In these simulations, the desired surge and heave velocities are set to $u_{d}=1 \mathrm{~m} / \mathrm{s}$ and $w_{d}=0 \mathrm{~m} / \mathrm{s}$. The bounds for $e$ and $U$ are taken as $|e| \leq 18 \mathrm{~m}$ and $0.5 \leq U \leq 1.2$. To ensure the asymptotic stability of the closed-loop control system, the control gains for the proposed controller are selected as $m_{e_{-} 11}=m_{11}, m_{e_{-} 33}=m_{33}, m_{e_{-} 55}=m_{55}$, $k_{11}=k_{12}=k_{13}=1, k_{21}=k_{22}=k_{23}=200 \quad$ and $k_{31}=k_{32}=k_{33}=100, k_{1}=0.085, k_{2}=0.35, k_{3}=2, k_{4}=2$ and $k_{5}=9$. Here the desired reference path of two scenarios are as follows: (1) a composite path that are constructed by the interconnection of three straight lines and a circle arc; (2) a sinusoidal path. 
Simulation results of following a composite path are provided in Figs. 3 and 4. Specifically, a simple observation of Fig. 3 (a) shows that, with the proposed controller, the AUV is capable of following the reference path even though perturbed by internal and external disturbances. Fig. 3 (b) illustrates that the cross-track error and path angle tracking error converge to a small neighborhood of the origin. It must be emphasized that the proposed method does not directly design $e$ and $\gamma_{e}$ to make all of the errors converge to a neighborhood of the origin that can be made arbitrarily small. Alternatively, a virtual desired pitching rate is constructed to represent the desired control objectives, making full use of the natural time-scale separation. Therefore, the proposed controller looks like much smarter. Also noted, the composite path includes two different cases: broken line and so called Dubins path. Slight tremble is unavoidable when desired pitching rate is suddenly changed due to transition from a line/arc to an arc/line. Referring to Fig. 3 (b), one might observe that the dynamic responses of the vehicle are very fast in those cases. The errors are sufficiently small and the good performance is guaranteed. Fig. 4 shows the estimation performance using the proposed ESO. Specifically, Fig. 4 (a) illustrates that the state variables $u, w$ and $q$ can be estimated by the developed ESO. The comparison of the given disturbances and the estimated disturbances are shown in Fig. 4 (b). It reveals that the unknown disturbances, adding on the closed-loop control system, also can be accurately estimated, using the proposed ESO, even though the velocity information feedback is unavoidable.

Moreover, without loss of generality of the proposed controller, a sinusoidal desired path is considered. The simulation results are provided in Figs. 5 and 6. Comparing the reference path with the actual result, a good path following ability of proposed controller is observed in the case of large initial tracking errors and unknown disturbances. Besides, the observation of Fig. 5 and 6 shows that the same analysis mentioned before also holds for this case.

In all of above simulation results, it is clear to see that the proposed controller can follow not only straight line but also curve path. The efficacy of the proposed approach to path following control of AUVs subject to internal and external disturbances is therefore sufficiently demonstrated.
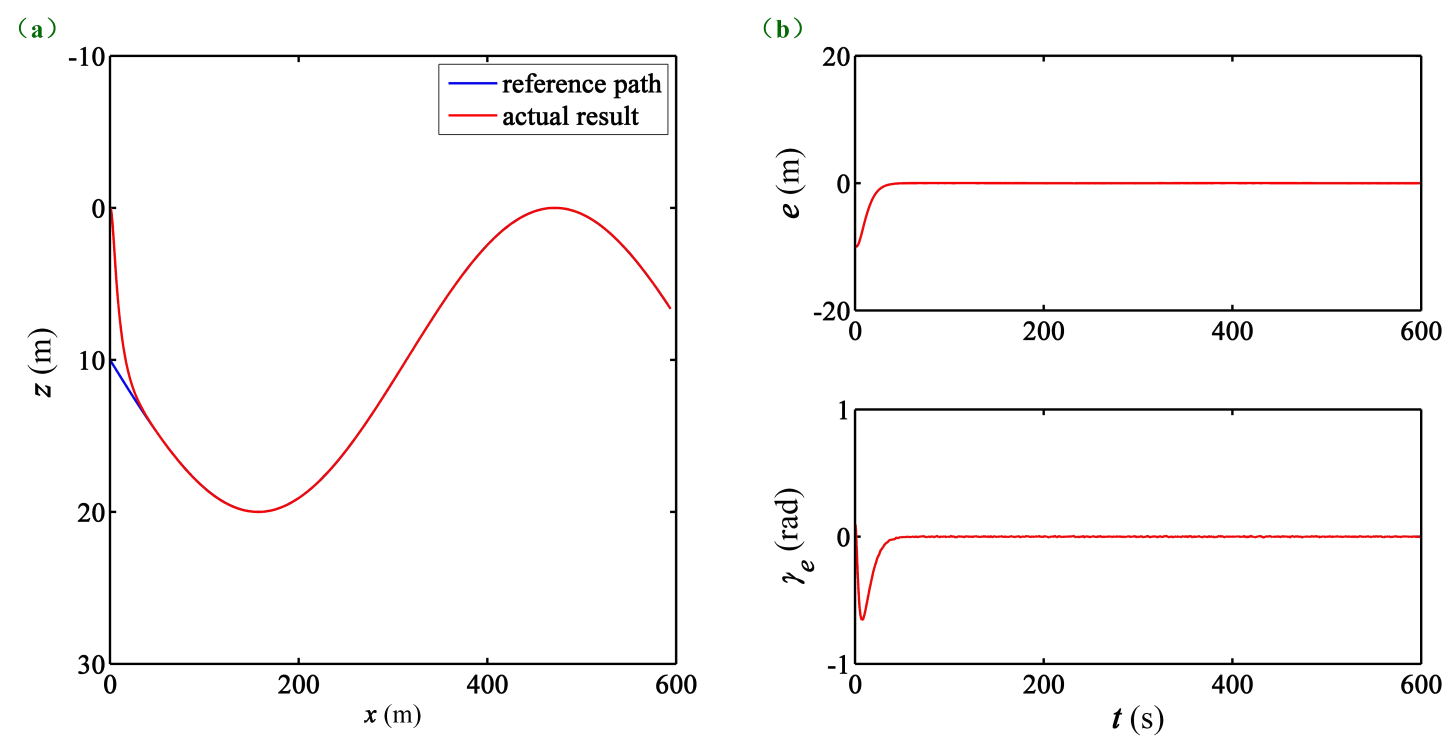

Fig. 5. Simulation results of following a sinusoidal path. (a) a composite path (blue solid line: reference path; red solid line: the actual result); (b) cross-track error and path angle tracking error. 

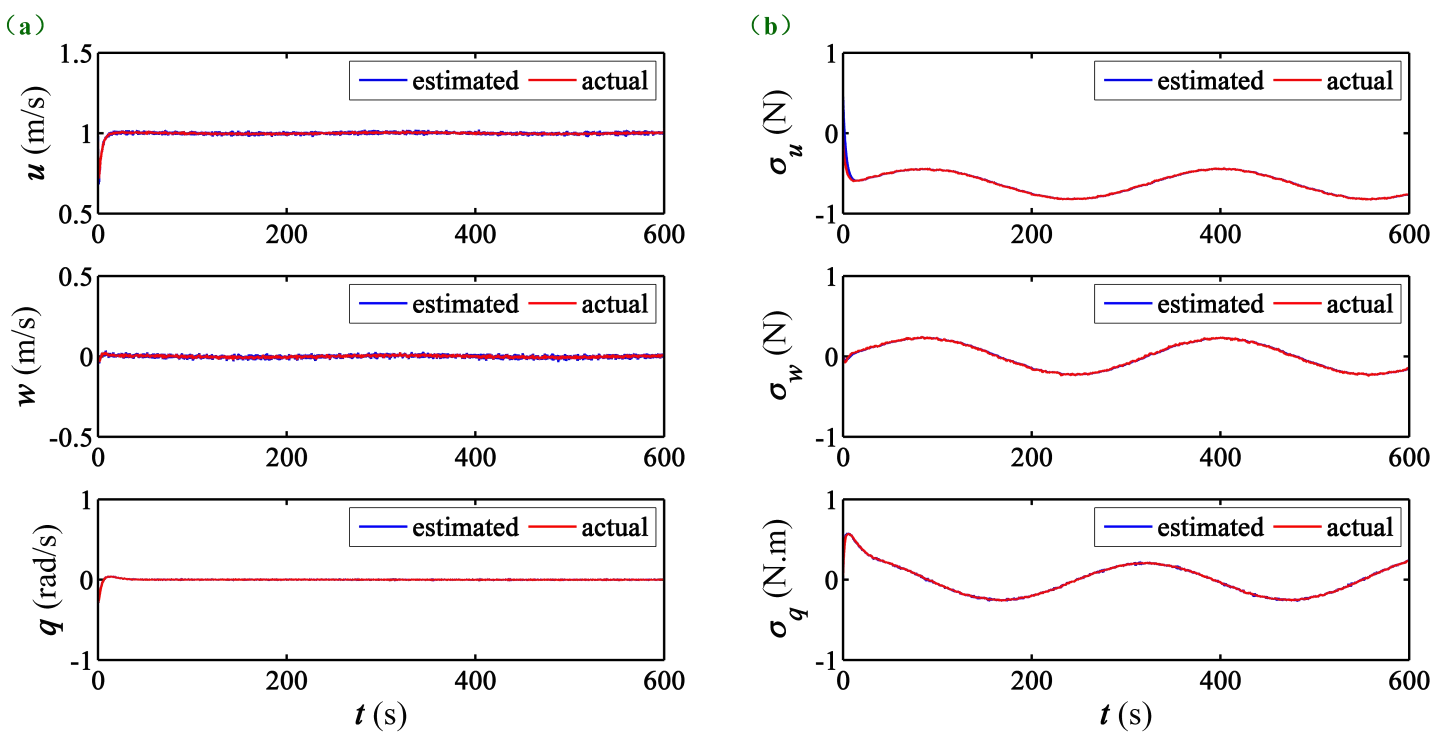

Fig. 6. Estimation performance by using the ESO

\section{Conclusion}

This paper considered the path following control problem of AUVs subject to unknown internal and external disturbances. The overall path following controller was divided into two terms: an ESO and a stabilizing controller. The proposed ESO was first given, in term of the singular perturbation theory. It must be emphasized that the asymptotic stability of the proposed ESO does not rely on the condition $\quad \dot{\sigma}=0$ as in the earlier study [28]. The stabilizing controller was devised based on the time scale decomposition method. Specifically, the stabilizing control system was considered as a three time scale singularly perturbed system. Singular perturbation theory was thus applied to analyze the system properties via order reduction. After the independent design of each subsystem, a simple stabilizing control law was given. Moreover, we proved the asymptotic stability of the closed-loop system with a composite Lyapunov function, which allowed to provide mathematical bounds of the control gains. Simulation studies were also conducted to evaluate the control performance and a good path following ability was observed. They revealed that the proposed controller can stabilize the vehicle to desired path under unknown internal and external disturbances.

\section{ACKNOWLEDGMENTS}

Conflict of Interest: The authors declare that they have no conflict of interest.

Funding: This study was funded by National Natural Science Foundation of China (grant number 51879057).

In addition, this paper is an original manuscript. There is no dual publication.

\section{APPENDIX}

Proof of assumption (1) - (4)

Recalling (27) and (30), we satisfy conditions (1) and (2) with $\alpha_{1}=2 k_{1} \hat{U} / \pi, \alpha_{2}=k_{2}, \varphi_{1}(\boldsymbol{x})=|e|$ and $\varphi_{2}(\tilde{\boldsymbol{y}})=\left|\tilde{\gamma}_{e}\right|$. Using these results, we compute 


$$
\begin{aligned}
& \quad \frac{\partial V_{2}(\boldsymbol{x})}{\partial \boldsymbol{x}}[f(\boldsymbol{x}, \boldsymbol{y}, H(\boldsymbol{x}, \boldsymbol{y}))-f(\boldsymbol{x}, G(\boldsymbol{x}), H(\boldsymbol{x}, G(\boldsymbol{x})))] \\
& =e\left[-\hat{U} \sin \left(\gamma_{e}\right)+\hat{U} \sin \left(\hat{\gamma}_{e}\right)\right] \\
& \leq \hat{U} \varphi_{1}(\boldsymbol{x}) \varphi_{21}(\tilde{\boldsymbol{y}})
\end{aligned}
$$

and

$$
\begin{aligned}
& {\left[\frac{\partial W_{2}(\boldsymbol{x}, \tilde{\boldsymbol{y}})}{\partial \boldsymbol{x}}-\frac{\partial W_{2}(\boldsymbol{x}, \tilde{\boldsymbol{y}})}{\partial \tilde{\boldsymbol{y}}} \frac{\partial G(\boldsymbol{x})}{\partial \boldsymbol{x}}\right] f(\boldsymbol{x}, \boldsymbol{y}, H(\boldsymbol{x}, \boldsymbol{y}))} \\
& =-k_{1} \tilde{\gamma}_{e} \hat{U} \sin \left(\gamma_{e}\right) \\
& \leq k_{1} \hat{U}\left|\tilde{\gamma}_{e}\right|\left(\left|\tilde{\gamma}_{e}\right|+k_{1}|e|\right) \\
& \leq k_{1} \hat{U}\left[k_{1} \varphi_{1}(\boldsymbol{x})+\varphi_{2}(\tilde{\boldsymbol{y}})\right] \varphi_{2}(\tilde{\boldsymbol{y}})
\end{aligned}
$$

thereby satisfy assumptions (3) and (4) with $\beta_{1}=\hat{U}, \quad \beta_{2}=k_{1}^{2} \hat{U}, \quad \beta_{3}=k_{1} \hat{U}$.

Proof of assumption (5) - (7)

Recalling (33), we satisfy the assumption (5) with $a_{2}=1, \varphi_{4}(\tilde{z})=\sqrt{k_{3} \tilde{u}_{e}^{2}+k_{4} \tilde{w}_{e}^{2}+k_{5} \tilde{\chi}_{e}^{2}}$. Following that,

$$
\begin{aligned}
& \frac{\partial\left[\left(1-n_{2}\right) V_{2}(\boldsymbol{x})+n_{2} W_{2}(\boldsymbol{x}, \tilde{\boldsymbol{y}})\right]}{\partial(\boldsymbol{x}, \tilde{\boldsymbol{y}})}\left[\begin{array}{c}
\Delta F \\
\Delta G
\end{array}\right] \\
& =\left(1-n_{2}\right) e\left[-U \sin \left(\gamma_{e}\right)+\hat{U} \sin \left(\gamma_{e}\right)\right]+n_{2} \tilde{\gamma}_{e}\left[\chi_{e}-\hat{\chi}_{e}\right] \\
& \leq\left(1-n_{2}\right) e\left(\left|\tilde{u}_{e}\right|+\left|\tilde{w}_{e}\right|\right)+n_{2} \tilde{\gamma}_{e} \tilde{\chi}_{e} \\
& \leq\left[\left(1-n_{2}\right)\left(\frac{1}{\sqrt{k_{3}}}+\frac{1}{\sqrt{k_{4}}}\right) \varphi_{1}(\boldsymbol{x})+n_{2} \frac{1}{\sqrt{k_{5}}} \varphi_{2}(\tilde{\boldsymbol{y}})\right] \varphi_{4}(\tilde{\boldsymbol{z}})
\end{aligned}
$$

and

$$
\begin{aligned}
& {\left[\frac{\partial W_{1}(\boldsymbol{x}, \boldsymbol{y}, \tilde{\boldsymbol{z}})}{\partial(\boldsymbol{x}, \boldsymbol{y})}-\frac{\partial W_{1}(\boldsymbol{x}, \boldsymbol{y}, \tilde{\boldsymbol{z}})}{\partial \tilde{\boldsymbol{z}}} \frac{\partial H(\boldsymbol{x}, \boldsymbol{y})}{\partial(\boldsymbol{x}, \boldsymbol{y})}\right]\left[\begin{array}{c}
\dot{\boldsymbol{x}} \\
\dot{\boldsymbol{y}}
\end{array}\right]} \\
& =k_{1} k_{2} \tilde{\chi}_{e} U \sin \left(\gamma_{e}\right)+k_{2} \tilde{\chi}_{e} \chi_{e} \\
& \leq k_{2}\left|\tilde{\chi}_{e}\right|\left[k_{1} U_{\max }\left(\left|k_{1} e\right|+\left|\tilde{\gamma}_{e}\right|\right)+k_{2}\left|\tilde{\gamma}_{e}\right|+\left|\tilde{\chi}_{e}\right|\right] \\
& \leq k_{2} \frac{1}{\sqrt{k_{5}}}\left[k_{1}^{2} U_{\max } \varphi_{1}(\boldsymbol{x})+\left(k_{1} U_{\text {max }}+k_{2}\right) \varphi_{2}(\tilde{\boldsymbol{y}})+\frac{1}{\sqrt{k_{5}}} \varphi_{4}(\tilde{z})\right] \varphi_{4}(\tilde{\boldsymbol{z}})
\end{aligned}
$$

Therefore, we satisfy the assumptions (6) and (7) with

$$
\begin{aligned}
& b_{1}=\left[\begin{array}{c}
\left(1-n_{2}\right)\left(1 / \sqrt{k_{3}}+1 / \sqrt{k_{4}}\right) \\
n_{2} / \sqrt{k_{5}}
\end{array}\right], \\
& b_{2}=\frac{k_{2}}{\sqrt{k_{5}}}\left[\begin{array}{c}
k_{1}^{2} U_{\max } \\
k_{1} U_{\max }+k_{2}
\end{array}\right], \\
& b_{3}=k_{2} / k_{5} .
\end{aligned}
$$




\section{REFERENCE}

1. Peymani, E., Fossen, T.I.: Path following of underwater robots using lagrange multipliers. Robotics \& Autonomous Systems, 67, 44-52, 2015.

2. Shen, C., Shi, Y., Buckham, B.: Trajectory tracking control of an autonomous underwater vehicle using Lyapunov-based model predictive control. IEEE Transactions on Industrial Electronics, 65(7), 5796-5805, 2017.

3. Peng, Z., Wang, J., Han, Q.L.: Path-following control of autonomous underwater vehicles subject to velocity and input constraints via neurodynamic optimization. IEEE Transactions on Industrial Electronics, 66(11), 8724-8732, 2018.

4. Peng, Z.H., Wang, J.: Output-Feedback path following Control of Autonomous Underwater Vehicles Based on an Extended State Observer and Projection Neural Networks. IEEE Transactions on Systems, Man, and Cybernetics: Systems, 48(4), 535-544, 2018.

5. Peng, Z.H., Wang, J.S., Wang, J.: Constrained Control of Autonomous Underwater Vehicles Based on Command Optimization and Disturbance Estimation. IEEE Transactions on Industrial Electronics, 66(5), 3627-3635, 2019.

6. Lapierre, L., Jouvencel, B.: Robust nonlinear path-following control of an AUV. IEEE Journal of Oceanic Engineering, 33(2), 89-102, 2008.

7. Park, B.S.: Adaptive formation control of underactuated autonomous underwater vehicles. Ocean Eng., 96, 1-7, 2015.

8. Elmokadem, T., Zribi, M., Youcef-Toumi, K.: Trajectory tracking sliding mode control of underactuated AUVs. Nonlinear Dynamics, 84(2), 1079-1091, 2016.

9. Xu, J., Wang, M., \& Qiao, L.: Dynamical sliding mode control for the trajectory tracking of underactuated unmanned underwater vehicles. Ocean Eng., 105, 54-63, 2015.

10. Joe, H., Kim,M., Yu, S.C.: Second-order sliding mode controller for autonomous underwater vehicle in the presence of unknown disturbances. Nonlinear Dynamics, 78(1), 183-196, 2014.

11. Qi, X.: Adaptive coordinated tracking control of multiple autonomous underwater vehicles. Ocean Engineering, 91, 84-90, 2014.

12. Do, K., Pan, J., Jiang, Z.: Robust and adaptive path following for underactuated autonomous underwater vehicles. Ocean Eng., 31(16), 1967-1997, 2004.

13. Li, J.H., Lee, P.M.: Design of an adaptive nonlinear controller for depth control of an autonomous underwater vehicle. Ocean Eng. 32(17), 2165-2181, 2005.

14. Xiang, X., Yu, C., Zhang, Q.: Robust fuzzy 3D path following for autonomous underwater vehicle subject to uncertainties. Comput. Oper. Res., 84, 165-177, 2017.

15. Salgado-Jimenez, T., Spiewak, J.M., Fraisse, P., Jouvencel, B.: A robust control algorithm for AUV: based on a high order sliding mode. In: Proceedings of the MTS/IEEE International Conference, OCEANS'04, Kobe, Japan, 276-281, 2004.

16. Aguiar, A.P., Hespanha, J.P.: Trajectory-tracking and path-following of underactuated autonomous vehicles with parametric modeling uncertainty. IEEE Transactions on Automatic Control, 52(8), 1362-1379, 2007.

17. Zhang, L.J., Qi, X., Pang, Y. J.: Adaptive output feedback control based on DRFNN for AUV. Ocean Eng., 36, 716-722, 2009. 
18. Fernandes, D., Sorensen, A.J., Pettersen, K.Y., Donha, D.C.: Output feedback motion control system for observation class ROVs based on a high-gain state observer: Theoretical and experimental results. Control Eng. Pract., 39, 90-102, 2015.

19. Khalil, H. K.: Nonlinear Systems. 3rd edition, Prentice Hall, Upper Saddle River, 2002.

20. Kokotovic, P., Khalil, H.K., O'reilly, J.: Singular Perturbation Methods in Control: Analysis and Design. ACADEMIC PRESS INC, 1999.

21. Canudas, d.W.C., Olguin, D.O.: Nonlinear control of an underwater vehicle/manipulator with composite dynamics. IEEE Transactions on Control Systems Technology, 8(6), 948-960, 2000.

22. Bhatta, P., Leonard, N.E.: Nonlinear Gliding Stability and Control for Vehicles with Hydrodynamic Forcing. Automatica, 44(5), 1240-1250, 2008.

23. Zhang, F., Tan, X.: Passivity-Based Stabilization of Underwater Gliders With a Control Surface. Journal of Dynamic Systems, Measurement, and Control, 137(6), 061006, 2015.

24. Ren, R.Y., Zou, Z.J., Wang, X.G.: A two-time scale control law based on singular perturbations used in rudder roll stabilization of ships. Ocean Eng., 88, 488-498, 2014.

25. Yi, B.W., Qiao, L., Zhang, W.D.: Two-time scale path following of under-actuated marine surface vessels: Design and stability analysis using singular perturbation methods. Ocean Eng., 124, 287- 297, 2016.

26. Prestero, T.: Verification of a Six-degree of Freedom Simulation Model for the REMUS Autonomous Underwater Vehicle. Ph.D. Thesis, Massachusetts Institute of Technology, 2001.

27. Lei, M.: Nonlinear diving stability and control for an AUV via singular perturbation. Ocean Eng., 197, 2020.

28. Chen, W.H., Ballance, Donald, J.: A nonlinear disturbance observer for robotic manipulators. IEEE Transactions on Industrial Electronics, 47(4), 932-938, 2000.

29. Jalving, B.: The NDRE-AUV flight control system. IEEE J. Ocean. Eng., 19(4), 497-501, 1994.

30. Fossen, T.I.: Guidance and Control of Ocean Vehicles. John Wiley and Sons., 1994

31. Lekkas, A.M., Fossen, T.I.: Line-of-Sight Guidance for Path Following of Marine Vehicles. In book: Advanced in Marine Robotics, Chapter 5, Lambert Academic Publishing., 2013.

32. Yurkevich, V.D.: PI/PID Control for Nonlinear Systems via Singular Perturbation Technique. Chapter from book: Advances in PID Control, DOI: 10.5772/19495, http://www.intechopen.com/books/advances-in-pid-control, 2011.

33. Naidu, A.S., Calise, A.J.: Singular Perturbations and Time Scales in Guidance and Control of Aerospace Systems: A Survey. Journal of Guidance, Control, and Dynamics, 24(6), 1057-1078., 2001.

34. Shinar, J.: On applications of singular perturbation techniques in nonlinear optimal control. Automatica, 19(2), 203-211, 1983.

35. Sheu, D., Vinh, N.X., Howe, R.M.: Application of singular perturbation methods for three-dimensional minimum-time interception. Journal of Guidance Control and Dynamics, 14(2), 360-367, 1991.

36. Esteban, S. Gordillo, F. Aracil, J.: Three-time scale singular perturbation control and stability analysis for an autonomous helicopter on a platform. Int. J. Robust Nonlinear Control, 23(12), 1360-1392, 2013.

37. Saberi, A., Khalil, H.K.: Quadratic-type Lyapunov functions for singularly perturbed systems. IEEE Trans. Automat. Contr., 29, 542-550, 1984.

38. Khalil, H.K.: Stability analysis of nonlinear multiparameter singularly perturbed systems. IEEE Transactions on Automatic Control, 32(3), 260-263, 1987. 
39. Pettersen, K., Egeland, O.: Time-varying exponential stabilization of the position and attitude of an underactuated autonomous underwater vehicle. IEEE Trans.Autom. Control, 44(1), 112-115, 1999. 
Figures

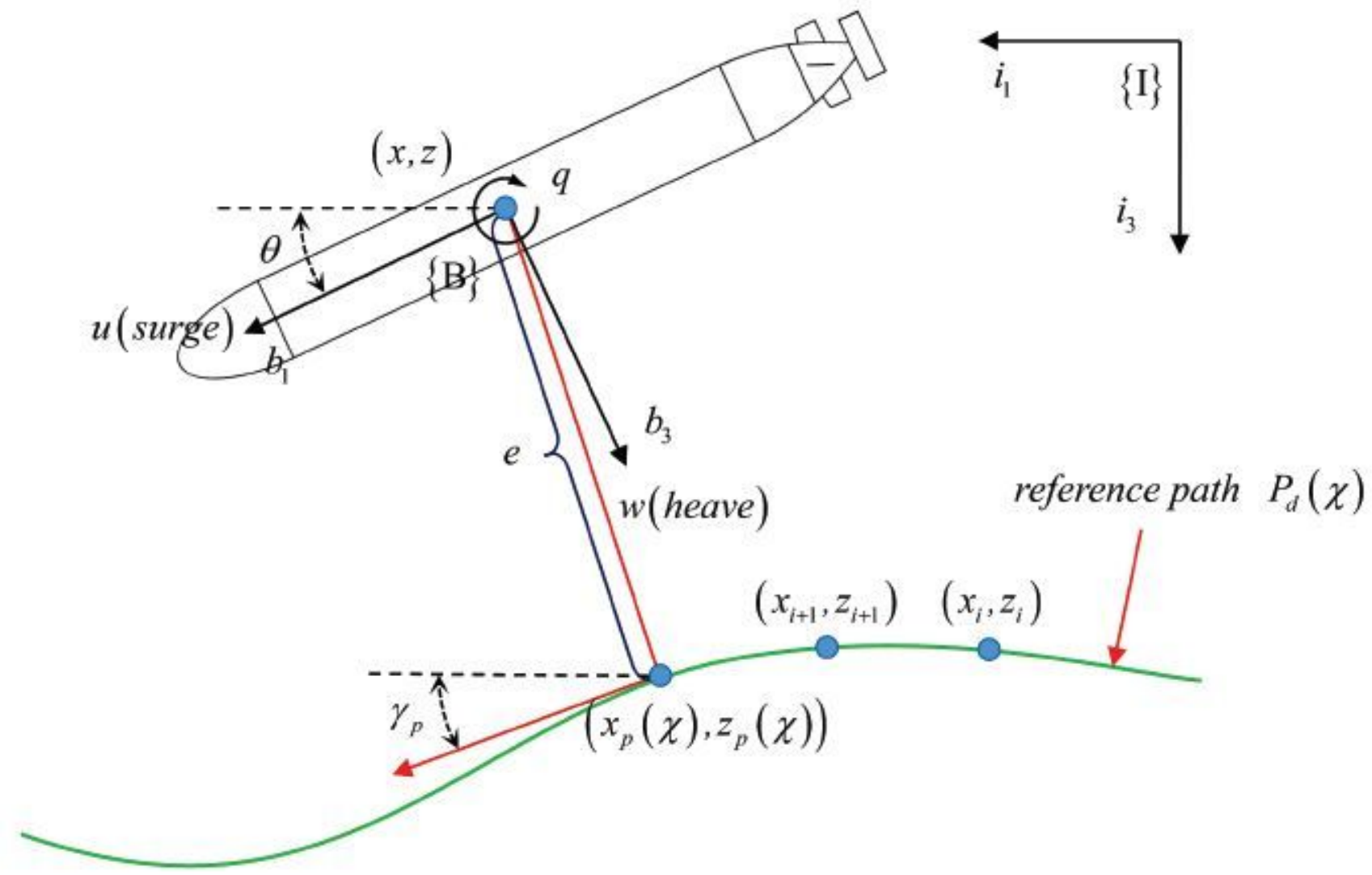

Figure 1

Vehicle model and reference frames.

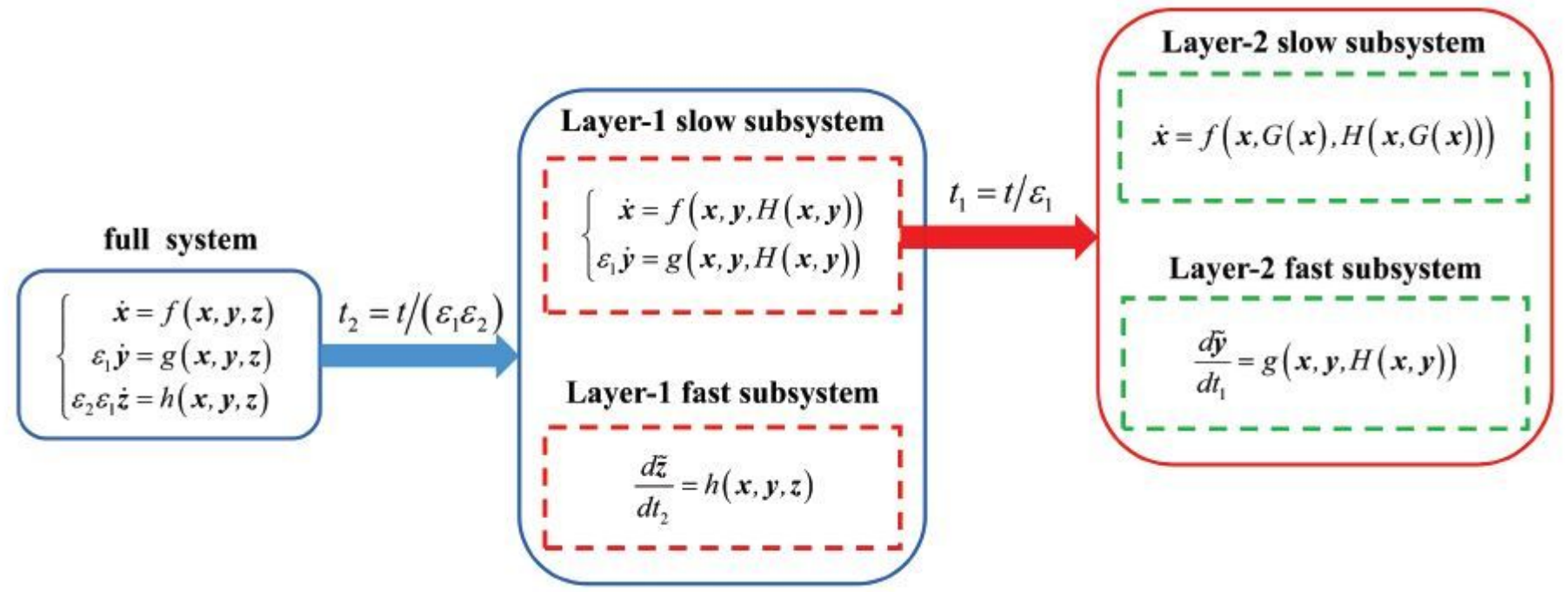

Figure 2 
Three-time scale singular perturbation reduction
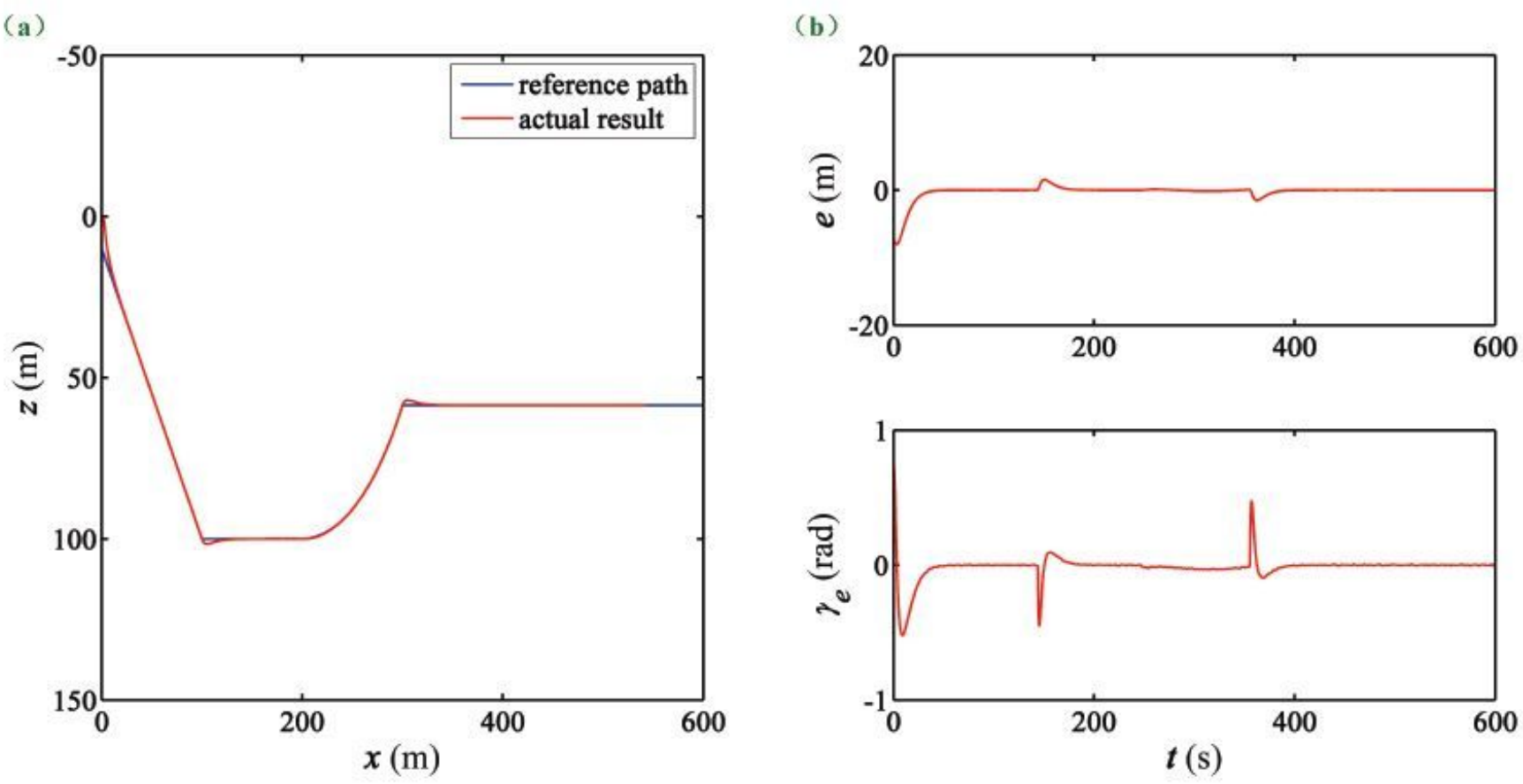

\section{Figure 3}

Simulation results of following a composite path. (a) a composite path (blue solid line: reference path; red solid line: the actual result); (b)cross-track errorand path angle tracking error.

(a)
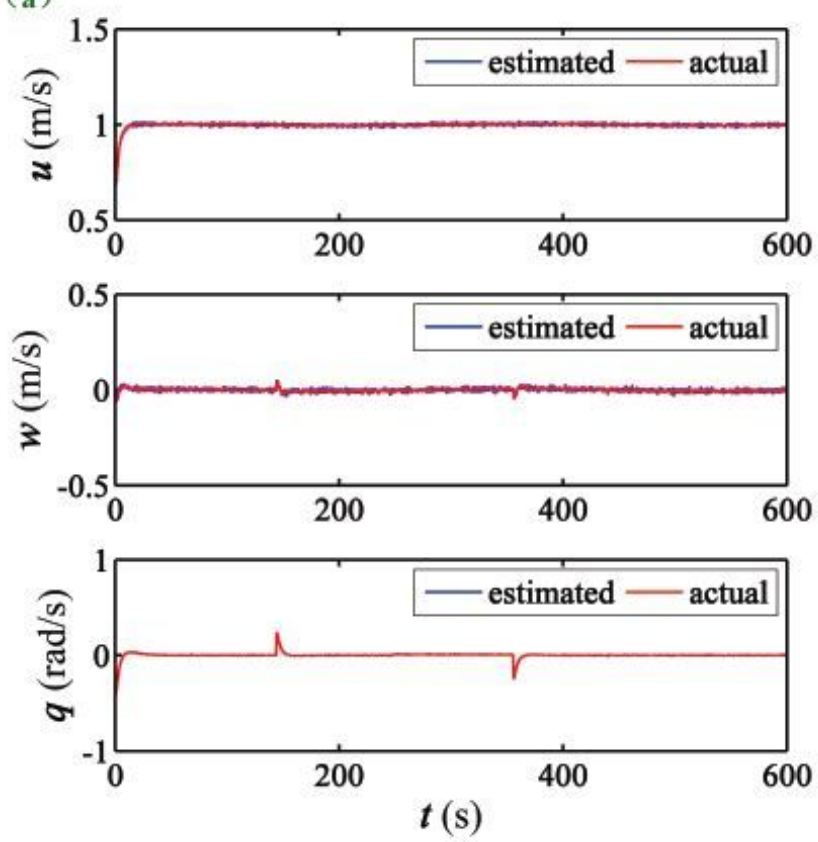

(b)
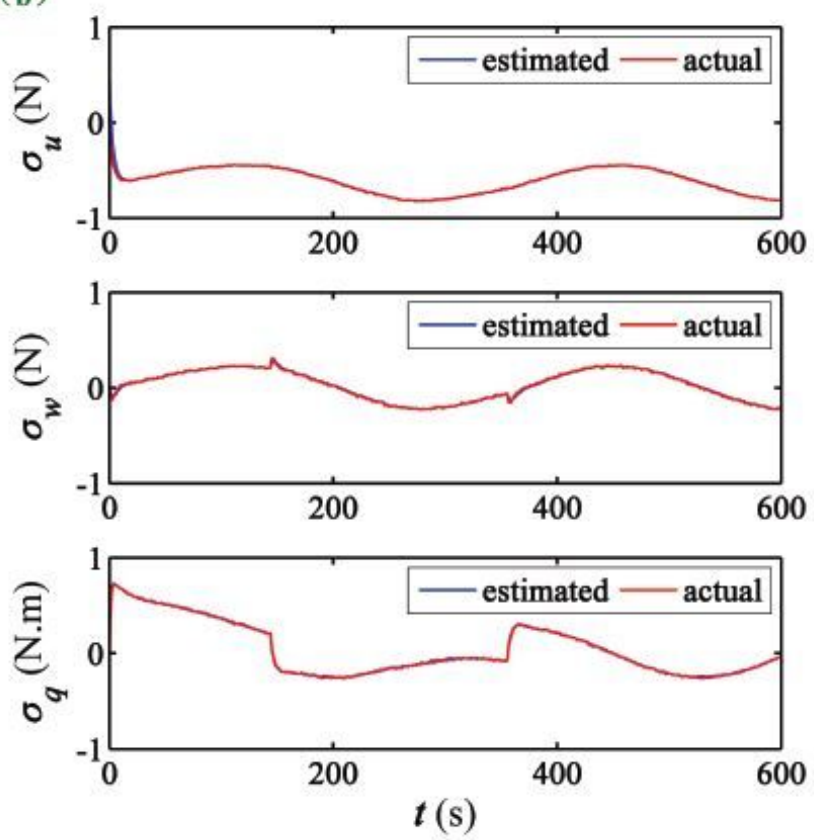

Figure 4 
Estimation performance by using the ESO.
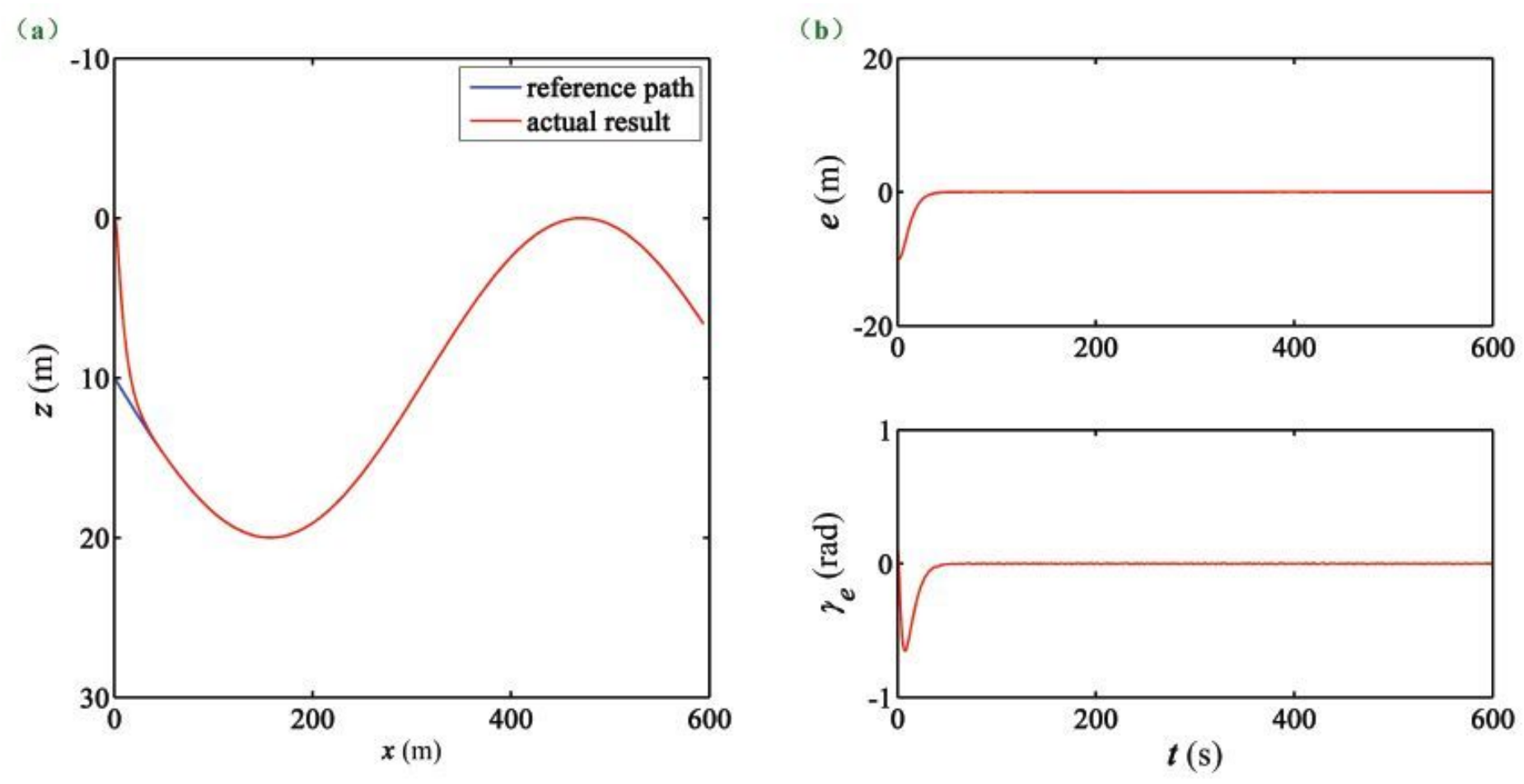

\section{Figure 5}

Simulation results of following a sinusoidal path. (a) a composite path (blue solid line: reference path; red solid line: the actual result); (b)cross-track errorand path angle tracking error.
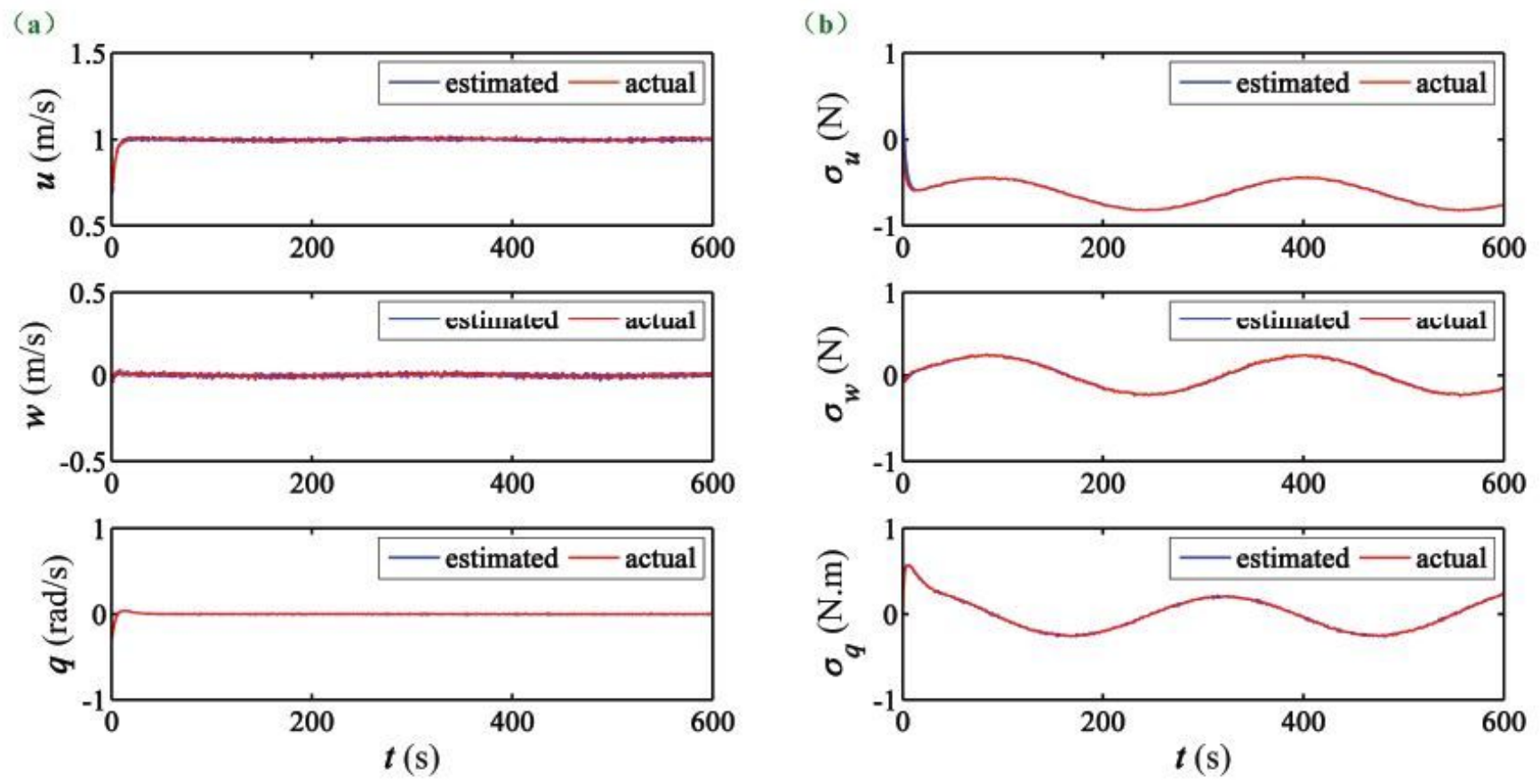

\section{Figure 6}

